\title{
Dissipative Analysis and Synthesis of Control for TS Fuzzy Markovian Jump Neutral Systems
}

\author{
R. Sakthivel, ${ }^{1,2}$ M. Rathika, ${ }^{3}$ and Srimanta Santra ${ }^{3}$ \\ ${ }^{1}$ Department of Mathematics, Sungkyunkwan University, Suwon 440 746, Republic of Korea \\ ${ }^{2}$ Department of Mathematics, Sri Ramakrishna Institute of Technology, Coimbatore, Tamil Nadu 641 010, India \\ ${ }^{3}$ Department of Mathematics, Anna University Regional Centre, Coimbatore, Tamil Nadu 641 047, India \\ Correspondence should be addressed to R. Sakthivel; krsakthivel0209@gmail.com
}

Received 17 February 2015; Accepted 2 May 2015

Academic Editor: Quanxin Zhu

Copyright (C) 2015 R. Sakthivel et al. This is an open access article distributed under the Creative Commons Attribution License, which permits unrestricted use, distribution, and reproduction in any medium, provided the original work is properly cited.

\begin{abstract}
This paper is focused on stochastic stability and strictly dissipative control design for a class of Takagi-Sugeno (TS) fuzzy neutral time delayed control systems with Markovian jumps. The main aim of this paper is to design a strictly dissipative controller such that the closed-loop TS fuzzy control system is stochastically stable, and also the disturbance rejection attenuation is obtained to a given level by means of the $H_{\infty}$ performance index. Intensive analysis is carried out to obtain sufficient conditions for the existence of desired dissipative controller which ensures both the stochastic stability and the strictly dissipative performance. The main advantage of the proposed technique is that it is possible to obtain the dissipative controller with less control effort and also, as special cases, robust $H_{\infty}$ control with the prescribed $H_{\infty}$ performance under given constraints and passivity control can be obtained for the considered systems. Also, the existence condition of the fuzzy dissipative controller can be obtained in terms of linear matrix inequalities. Finally, a practical example based on truck-trailer model is provided to demonstrate the effectiveness and feasibility of the proposed design technique.
\end{abstract}

\section{Introduction}

The Takagi-Sugeno (TS) fuzzy model is an effective one to analyze and synthesize nonlinear systems which are ubiquitous in signal processing, communications, chemical processes, robotics systems, and automotive systems [1-3]. In recent years, fuzzy control systems have become an important topic in systems theory due to their potential applications in many fields of science and engineering $[4,5]$. More precisely, Takagi-Sugeno (TS) fuzzy model based control plays an important role which offers a systematic and effective platform for control of nonlinear plants $[6,7]$. Also, with the rapid development of LMI techniques and Lyapunov stability theory, many important and interesting results have been reported on control of TS fuzzy systems [8-10]. By solving some convex optimization problems with LMI constraints, some effective $H_{\infty}$ filter designs are presented for discretetime Takagi-Sugeno fuzzy time-varying delay systems [11].

On the other hand, in many practical systems, the abrupt phenomena cannot be ignored and lead to the changes of system parameters [12-14]. On the other hand, it is well known that the systems with Markovian jump parameters can be used to model some practical systems where they may experience abrupt changes in their structure and parameters due to random failures, repairs of components, and sudden environmental disturbances [15-17]. These abrupt variations can be described by Markovian jump systems and hence the study of time-delay systems with Markovian jumps has received much attention [18-20]. Wang et al. [21] studied the problem of robust $H_{\infty}$ fuzzy control for a class of uncertain nonlinear Markovian jump systems with time-varying delay with use of a delay decomposition approach together with linear matrix inequalities.

The existence of time delays brings negative effects such as instability, oscillation, and poor performance to the dynamic performance of TS fuzzy systems [22-24]. Time-varying delay especially has an adverse impact not only on the system performance but also on its stability, therefore neglecting the effects of time-varying delay in system dynamics may lead to poor performance and instability $[25,26]$. A number 
of delay-dependent stabilization results for TS fuzzy systems have been studied and also effective results have been reported to reduce the conservatism for further improving the quality of delay-dependent stabilization criteria [27, 28]. Peng and Han [29] discussed the robust stabilization for a class of TS fuzzy control systems with interval time-varying delays. Li et al. [30] proposed a fuzzy state feedback controller which guarantees that the nonlinear time-delay singular Markovian jump system with partly unknown transition rates not only is regular, impulse-free, and stochastically stable, but also satisfies a prescribed $H_{\infty}$ performance for all delays.

Furthermore, the notion of dissipativity originated from circuit analysis [31, 32] is a generalization of the passivity which plays an important role in system and control theory both from theoretical and practical points of view. In the past two decades, there have been considerable interests in the analysis and synthesis of dissipative control for dynamical systems [33, 34]. Feng and Lam [35] investigated the problem of reliable dissipative control for a continuous-time singular Markovian system with actuator failure and also in which a new set of sufficient conditions is established in terms of linear matrix inequalities to ensure that a singular Markovian system is stochastically admissible and strictly dissipative. More recently, the problems of dissipativity analysis and synthesis for discrete-time Takagi-Sugeno fuzzy systems with stochastic perturbation and time-varying delay are discussed in [36]. Moreover, the study of neutral systems has received considerable attention during the past few decades because the system involves the derivative in the delayed state $[37,38]$.

On the other hand, the notion of dissipativity can be regarded as a generalization of $H_{\infty}$ performance as well as positive realness performances and passivity. Due to the importance of TS fuzzy neutral models, the development of dissipativeness analysis and dissipative control for neutral TS fuzzy systems became an essential and attractive topic. However, to the best of our knowledge, the dissipative control problem for a class of TS fuzzy model Markovian jump neutral systems with time-varying delay has not been fully investigated yet. Motivated by this consideration, in this paper, we investigate the dissipative control problem while satisfying a prescribed disturbance attenuation level for a class of continuous time Markovian jump neutral systems which is described by TS fuzzy model with time-varying delay. Based on the obtained LMI conditions, the solvable conditions for the existence of dissipative controller are derived which guarantee that the closed-loop system is not only stochastically stable but also strictly dissipative for all admissible uncertainties. It is worth pointing out that the dissipative control problem considered here includes the $H_{\infty}$ control problem, passivity based control problem, and mixed $H_{\infty}$ and passivity problem as special cases. In order to obtain the required result, an appropriate novel Lyapunov functional containing four integral terms involving the upper bounds of the delay is proposed. An attractive feature of the employed Lyapunov-Krasovskii functional is that it can effectively deal with the dissipativity of neutral TS-fuzzy systems with Markovian jumping parameters. Further, the results reveal that it is possible to obtain the dissipative controller with less control effort. Finally, a numerical example is provided to illustrate the effectiveness of the method proposed in this paper.

Notations. The superscripts " $T$ " and " $(-1)$ " stand for matrix transposition and matrix inverse, respectively; $R^{n}$ and $R^{n \times m}$ denote the $n$-dimensional Euclidean space and the set of all $n \times m$ real matrices, respectively; $X \geq Y$ (resp., $X>Y$ ), where $X$ and $Y$ are symmetric matrices, means that $X-Y$ is a positive semidefinite (resp., positive definite); $I$ is the identity matrix of appropriate dimension; $\mathscr{L}_{2}[0, \infty)$ is the space of square integrable function over $[0, \infty) ;\left(\Omega, \mathscr{F},\left(\mathscr{F}_{t}\right)_{t>0}, \mathscr{P}\right)$ is a complete probability space with filtration $\left(\mathscr{F}_{t}\right)_{t>0}$, where $\Omega$ is the sample space, $\mathscr{F}$ is the $\sigma$-algebra of subsets of the sample space, and $\mathscr{P}$ is the probability measure on $\mathscr{F}$. The notation $\mathbb{E}[\cdot]$ stands for the expectation operator; and "*" is used to represent a term that is induced by symmetry.

\section{Problem Formulation and Preliminaries}

Consider a class of continuous time-delay Markovian jump systems in the probability space $\left(\Omega, \mathscr{F},\left\{\mathscr{F}_{t}\right\}_{t \geq 0}, \mathscr{P}\right)$ that is described by the TS fuzzy model. The $i$ th rule of TS fuzzy model is of the following form.

$$
\begin{aligned}
& \text { Plant Rule } i \text {. If } \mu_{1}(t) \text { is } F_{1}^{i}, \mu_{2}(t) \text { is } F_{2}^{i} \text {, and } \ldots, \mu_{g}(t) \text { is } F_{g}^{i} \text {, then } \\
& \qquad \begin{aligned}
\dot{x}(t)= & A_{i}\left(r_{t}\right) x(t)+A_{d i}\left(r_{t}\right) x(t-\tau(t)) \\
& +J_{i}\left(r_{t}\right) \dot{x}(t-h(t))+B_{i}\left(r_{t}\right) u(t) \\
& +B_{1 i}\left(r_{t}\right) \omega(t), \\
z(t)= & C_{i}\left(r_{t}\right) x(t)+D_{i}\left(r_{t}\right) u(t), \\
x(t)= & \phi(t), \\
& r_{t}=r_{0}, t \in[-d, 0], i=1,2, \ldots, \mathbb{S},
\end{aligned}
\end{aligned}
$$

where $x(t) \in R^{n}$ is the state vector; $u(t) \in R^{m}$ is the control input; $\omega(t) \in \mathscr{L}_{2}^{q}[0, \infty)$ denotes the external disturbances; $z(t) \in R^{p}$ is the control output; $\phi(t)$ is a continuous vectorvalued initial function defined on $\mathscr{L}_{2}^{n}[-d, 0]$; and $r_{0}$ is the initial mode. Further, $\mu_{1}(t), \mu_{2}(t), \ldots, \mu_{g}(t)$ are the premise variables that depend on the states in many cases; $F_{l}^{i}, i=$ $1,2, \ldots, \mathbb{S}, l=1,2, \ldots, g$, are the fuzzy sets; $\mathbb{S}$ is the number of IF-THEN rules; $\left\{r_{t}, t \geq 0\right\}$ is a continuous-time discrete state Markovian process with right continuous values in a finite set $\mathbb{M}=\{1,2, \ldots, N\} ; A_{i}\left(r_{t}\right), A_{d i}\left(r_{t}\right), J_{i}\left(r_{t}\right), B_{i}\left(r_{t}\right), B_{1 i}\left(r_{t}\right)$, $C_{i}\left(r_{t}\right)$, and $D_{i}\left(r_{t}\right)$ are known mode-dependent real constant matrices with appropriate dimensions for each $r_{t} \in \mathbb{M}$. Also, the transition probability matrix $\Pi=\pi_{r k}(r, k \in \mathbb{M})$ is given by

$$
\begin{aligned}
\mathscr{P} & \left\{r_{t+\delta(t)}=k \mid r_{t}=r\right\} \\
& = \begin{cases}\pi_{r k} \delta(t)+O(\delta(t)) & \text { if } k \neq r, \\
1+\pi_{k k} \delta(t)+O(\delta(t)) & \text { if } k=r,\end{cases}
\end{aligned}
$$

where $\delta(t)>0$ and $\lim _{\delta(t) \rightarrow 0}(O(\delta(t)) / \delta(t))=0$ and $\pi_{r k} \geq 0$ is the transition rate from mode $k$ at time $t$ to mode $r$ at time $t+\delta(t)$ if $k \neq r$ and $\pi_{r r}=-\sum_{k=1, k \neq r}^{N} \pi_{r k}$. 
Also, in this paper, we consider time-varying continuous functions $\tau(t)$ and $h(t)$ that satisfy for all $t \geq 0$

$$
\begin{array}{ll}
0<\tau(t) \leq \bar{\tau}<\infty, & \dot{\tau}(t) \leq \bar{\tau}_{1}<1, \\
0<h(t) \leq \bar{h}<\infty, & \dot{h}(t) \leq \bar{h}_{1}<1,
\end{array}
$$

where $\bar{\tau}=\max \{\tau(t)\}, \bar{h}=\max \{h(t)\}$, and $d=\max \{\bar{\tau}, \bar{h}\}$. For notational simplicity, take $r_{t}=r, r \in \mathbb{M}$; a matrix $J_{i}\left(r_{t}\right)$ will be denoted by $J_{i}(r)$, and $A_{i}\left(r_{t}\right)$ is denoted by $A_{i}(r), A_{d i}\left(r_{t}\right)$ by $A_{d i}(r)$, and so on.

By using a singleton fuzzifier, a center average defuzzifier, and product inference, the final state and output of fuzzy neutral Markovian jump system (1) can be expressed as

$$
\begin{aligned}
\dot{x}(t) & =\sum_{i=1}^{\mathbb{S}} h_{i}(\mu(t))\left[A_{i}(r) x(t)+A_{d i}(r) x(t-\tau(t))\right. \\
\left.+J_{i}(r) \dot{x}(t-h(t))+B_{i}(r) u(t)+B_{1 i}(r) \omega(t)\right] & \\
z(t) & =\sum_{i=1}^{\mathbb{S}} h_{i}(\mu(t))\left[C_{i}(r) x(t)+D_{i}(r) u(t)\right] \\
x(t) & =\phi(t)
\end{aligned}
$$$$
r=r_{0}, t \in[-d, 0], i=1,2, \ldots, \mathbb{S}
$$

where $h_{i}(\mu(t))=w_{i}(\mu(t)) / \sum_{i=1}^{\mathbb{S}} w_{i}(\mu(t)), w_{i}(\mu(t))=$ $\prod_{l=1}^{g} F_{l}^{i}\left(\mu_{l}(t)\right)$, in which $F_{l}^{i}\left(\mu_{l}(t)\right)$ is the grade of membership of $\mu_{l}(t)$ corresponding to the fuzzy set $F_{l}^{i}$, and $\mu(t)=$ $\left[\mu_{1}(t), \mu_{2}(t), \ldots, \mu_{g}(t)\right]$. It is assumed that $w_{i}(\mu(t)) \geq 0$ and $\sum_{i=1}^{\mathbb{S}} w_{i}(\mu(t))>0$; then we can get that $\sum_{i=1}^{\mathbb{S}} w_{i}(\mu(t))=1$; $0 \leq w_{i}(\mu(t)) \leq 1, i=1,2, \ldots, \mathbb{S}$.

Also, by adapting the idea discussed in [39], for the fuzzy neutral Markovian jump system (1), we construct the state feedback controller in the following form.

\section{Controller Part}

Rule $i$. If $\mu_{1}(t)$ is $F_{1}^{i}, \mu_{2}(t)$ is $F_{2}^{i}$, and $\ldots, \mu_{g}(t)$ is $F_{g}^{i}$, then

$$
u(t)=K_{i}(r) x(t), \quad i=1,2, \ldots, \mathbb{S},
$$

where $K_{i}(r)$ is the state feedback gain to be determined. By incorporating the fuzzy rule, the state feedback control law can be written as

$$
u(t)=\sum_{i=1}^{\mathbb{S}} h_{i}(\mu(t)) K_{i}(r) x(t) .
$$

Substituting (6) in (4), we can be obtain the closed-loop fuzzy Markovian jump control system in the form

$$
\begin{aligned}
\dot{x}(t)= & \widehat{A}(r) x(t)+\widehat{A}_{d}(r) x(t-\tau(t)) \\
& +\widehat{J}(r) \dot{x}(t-h(t))+\widehat{B}_{1}(r) \omega(t), \\
z(t)= & \widehat{C}(r) x(t), \\
x(t)= & \phi(t), \\
& r=r_{0}, t \in[-d, 0], i=1,2, \ldots, \mathbb{S},
\end{aligned}
$$

where

$$
\begin{aligned}
& \widehat{A}(r) \\
& =\sum_{i=1}^{\mathbb{S}} h_{i}(\mu(t)) \sum_{j=1}^{\mathbb{S}} h_{j}(\mu(t))\left[A_{i}(r)+B_{i}(r) K_{j}(r)\right], \\
& \widehat{A}_{d}(r)=\sum_{i=1}^{\mathbb{S}} \sum_{j=1}^{\mathbb{S}} h_{i}(\mu(t)) A_{d i}(r), \\
& \widehat{J}(r)=\sum_{i=1}^{\mathbb{S}} \sum_{j=1}^{\mathbb{S}} h_{i}(\mu(t)) J_{i}(r), \\
& \widehat{B_{1}}(r)=\sum_{i=1}^{\mathbb{S}} \sum_{j=1}^{\mathbb{S}} h_{i}(\mu(t)) B_{1 i}(r), \\
& \widehat{C}(r) \\
& \quad=\sum_{i=1}^{\mathbb{S}} h_{i}(\mu(t)) \sum_{j=1}^{\mathbb{S}} h_{j}(\mu(t))\left[C_{i}(r)+D_{i}(r) K_{j}(r)\right] .
\end{aligned}
$$

Definition 1. Consider $V\left(x_{t}, r_{t}, t\right)$ as the stochastic lyapunov function of the resulting system (1); its weak infinitesimal operator is defined as

$$
\begin{aligned}
& £ V\left(x_{t}, r_{t}, t\right) \\
& \quad=\lim _{\Delta t \rightarrow 0} \frac{1}{\Delta t}\left[\mathbb{E}\left\{V\left(x_{t+\Delta t}, r_{t+\Delta t}, t+\Delta t\right)-V\left(x_{t}, r_{t}, t\right)\right\}\right] .
\end{aligned}
$$

Definition 2. The fuzzy Markovian jump time-delay neutral system (7) is said to be stochastically stable if there exists a scalar $M\left(r_{0}, \phi(\cdot)\right)$ such that

$$
\begin{aligned}
& \lim _{T \rightarrow \infty} \mathbb{E}\left\{\int_{0}^{T}\|x(t)\|^{2} d t \mid r_{0}, x(s)=\phi(s)\right\} \\
& \leq M\left(r_{0}, \phi(\cdot)\right) .
\end{aligned}
$$

Definition 3. Given a scalar $\delta>0$, real matrices $Q=Q^{T}, R=$ $R^{T}$ and matrix $S$, the fuzzy Markovian jump neutral system (7) is strictly $(Q, S, R)$ dissipative, if for any $T \geq 0$, under zero initial state, the following condition is satisfied:

$$
\mathbb{E}\left[\langle z, Q z\rangle_{T}+2\langle z, S \omega\rangle_{T}+\langle\omega, R \omega\rangle_{T}\right] \geq \delta\langle\omega, \omega\rangle_{T},
$$

where the notation $\langle z, Q z\rangle_{T}$ represents $\int_{0}^{T} z^{T}(t) Q z(t) d t$ and the other symbols $(Q, S, R)$ are similarly defined. Also, we assume that $Q<0$ and $\bar{Q}=\sqrt{-Q}$.

Remark 4. Based on Definition 3, it can be seen that the above strict dissipativeness includes the following special cases:

(i) If $Q=-I, S=0$, and $R=\gamma^{2} I$, the strict $(Q, S, R)$ dissipativity reduces to the $H_{\infty}$ performance constraint.

(ii) If $Q=0, S=I$, and $R=0$, the strict $(Q, S, R)$ dissipativity reduces to a passivity performance. 
(iii) If $Q=-\theta I, S=(1-\theta) I$ and $R=\theta \gamma^{2} I$ or $Q=-\gamma^{-1} \theta I$, $S=(1-\theta) I$ and $R=\gamma \theta I$ where $\theta \in[0,1]$ is a given scalar weight representing a trade off between $H_{\infty}$ and passivity performance, then strictly $(Q, S, R)$ dissipativity reduces to the mixed $H_{\infty}$ and passivity performance.

Lemma 5 (see [40]). For any constant matrix $Z>0$, any scalars $a$ and $b$ with $a<b$, and $a$ vector function $x(t)$ : $[a, b] \rightarrow \mathbb{R}^{n}$ such that the integrals concerned are well defined, then the following holds:

$$
\begin{gathered}
{\left[\int_{a}^{b} x(s) d s\right]^{T} Z\left[\int_{a}^{b} x(s) d s\right]} \\
\quad \leq(b-a) \int_{a}^{b} x^{T}(s) Z x(s) d s .
\end{gathered}
$$

\section{Dissipativity Analysis}

In this section, the dissipative control problem is studied for a class of TS fuzzy neutral systems with Markovian jumps. First, we discuss the stochastic stability and dissipative conditions in the mean square sense and subsequently the result is extended to obtain the desired dissipative controller. More precisely, by assuming that the control gain $K_{j}$ is known, we will develop the condition in the following theorem in which the closed-loop system (7) is stochastically stable and strictly $(Q, S, R)$ dissipative.

Theorem 6. For the given scalars $\delta>0, \bar{\tau}_{1}>0, \bar{h}_{1}>0$, the matrices $Q=Q^{T}, S, R=R^{T}$ and the given control gain matrix $K_{j}$, the closed-loop system (7) is stochastically stable and strictly $(Q, S, R)$ dissipative, if there exist matrices $P(r)>0, Q_{1}(r)>0$, $Q_{i}>0, i=2,3, S_{i}(r)>0, R_{i}(r)>0, Z_{i}(r)>0, i=1,3, S_{j}>0$, $R_{j}>0, Z_{j}>0, j=2,4$, and any appropriate dimension matrices $M_{i}, i=1,2$, such that the following conditions hold for each $r \in \mathbb{M}$ :

$$
\begin{gathered}
\sum_{k=1}^{N} \pi_{r k} Q_{1}(k)-Q_{2}<0, \\
\sum_{k=1}^{N} \pi_{r k} S_{1}(k)-S_{2}<0, \\
\sum_{k=1}^{N} \pi_{r k} S_{3}(k)-S_{4}<0, \\
\sum_{k=1}^{N} \pi_{r k} R_{1}(k)-R_{2}<0, \\
\sum_{k=1}^{N} \pi_{r k} R_{3}(k)-R_{4}<0,
\end{gathered}
$$

$$
\begin{aligned}
& \sum_{k=1}^{N} \pi_{r k} Z_{1}(k)-Z_{2}<0, \\
& \sum_{k=1}^{N} \pi_{r k} Z_{3}(k)-Z_{4}<0, \\
& \Pi_{i i}<0, \quad i=1,2, \ldots, \mathbb{S}, \\
& \Pi_{i j}+\Pi_{j i}<0, \quad i<j, \quad i=j=1,2, \ldots, \mathbb{S},
\end{aligned}
$$

where $\Pi_{i j}=\left[\Pi_{i j}\right]_{m \times n}, m, n=1,2, \ldots, 9$, with

$$
\begin{aligned}
& \Pi_{i j_{1,1}}=Q_{1}(r)+\bar{\tau} Q_{2}+\sum_{k=1}^{N} \pi_{r k} P(k)+\bar{\tau} S_{1}(r)+\frac{\bar{\tau}^{2}}{2} S_{2} \\
& +\bar{h} S_{3}(r)+\frac{\bar{h}^{2}}{2} S_{4}-\frac{1}{\bar{\tau}} R_{1}(r)-\frac{1}{\bar{h}} R_{3}(r) \\
& -\operatorname{sym}\left(Z_{1}(r)\right)-\operatorname{sym}\left(Z_{3}(r)\right) \\
& +\operatorname{sym}\left(M_{1} \widehat{A}(r)\right)-\widehat{C}^{T}(r) Q \widehat{C}(r), \\
& \Pi_{i j_{1,2}}=M_{1} \widehat{A}_{d}(r) \text {, } \\
& \Pi_{i j_{1,3}}=\frac{1}{\bar{\tau}} R_{1}(r), \\
& \Pi_{i j_{1,4}}=\frac{1}{\bar{h}} R_{3}(r) \text {, } \\
& \Pi_{i j_{1,5}}=P(r)-M_{1}+\widehat{A}^{T}(r) M_{2}^{T}, \\
& \Pi_{i j_{1,6}}=M_{1} \widehat{J}(r) \text {, } \\
& \Pi_{i j_{1,7}}=\frac{1}{\bar{\tau}}\left(Z_{1}(r)+Z_{1}^{T}(r)\right), \\
& \Pi_{i j_{1,8}}=\frac{1}{\bar{h}}\left(Z_{3}(r)+Z_{3}^{T}(r)\right), \\
& \Pi_{i j_{1,9}}=M_{1} \widehat{B}_{1}(r)-\widehat{C}^{T}(r) S \text {, } \\
& \Pi_{i j_{2,2}}=-\left(1-\bar{\tau}_{1}\right) Q_{1}(r) \text {, } \\
& \Pi_{i j_{2,5}}=\widehat{A}_{d}^{T}(r) M_{2}^{T} \text {, } \\
& \Pi_{i j_{3,3}}=-\frac{1}{\bar{\tau}} R_{1}(r) \text {, } \\
& \Pi_{i j_{4,4}}=-\frac{1}{\bar{h}} R_{3}(r) \text {, } \\
& \Pi_{i j_{5,5}}=Q_{3}+\bar{\tau} R_{1}(r)+\frac{\bar{\tau}^{2}}{2} R_{2}+\bar{h} R_{3}(r)+\frac{\bar{h}^{2}}{2} R_{4} \\
& +\frac{\bar{\tau}^{2}}{2} Z_{1}(r)+\frac{\bar{\tau}^{3}}{6} Z_{2}+\frac{\bar{h}^{2}}{2} Z_{3}(r)+\frac{\bar{h}^{3}}{6} Z_{4} \\
& -\operatorname{sym}\left(M_{2}\right) \text {, }
\end{aligned}
$$




$$
\begin{aligned}
& \Pi_{i j_{5,6}}=M_{2} \widehat{J}(r), \\
& \Pi_{i j_{5,9}}=M_{2} \widehat{B}_{1}(r), \\
& \Pi_{i j_{6,6}}=-\left(1-\bar{h}_{1}\right) Q_{3}, \\
& \Pi_{i j_{7,7}}=-\frac{1}{\bar{\tau}} S_{1}(r)-\frac{1}{\bar{\tau}^{2}} \operatorname{sym}\left(Z_{1}(r)\right), \\
& \Pi_{i j_{8,8}}=-\frac{1}{\bar{h}} S_{3}(r)-\frac{1}{\bar{h}^{2}} \operatorname{sym}\left(Z_{3}(r)\right), \\
& \Pi_{i j_{9,9}}=-(R-\delta I)
\end{aligned}
$$

and the remaining parameters are zero.

Proof. In order to obtain the required result, we construct the Lyapunov-Krasovskii functional (LKF) candidate for system (7) in the following form:

$$
V_{i}(x(t), r, t)=\sum_{i=1}^{8} V_{i}(x(t), r, t),
$$

where

$$
\begin{aligned}
& V_{1}(x(t), r, t)=x^{T}(t) P(r) x(t), \\
& V_{2}(x(t), r, t) \\
& =\int_{t-\tau(t)}^{t} x^{T}(s) Q_{1}(r) x(s) d s \\
& +\int_{-\tau(t)}^{0} \int_{t+\theta}^{t} x^{T}(s) Q_{2} x(s) d s d \theta \\
& +\int_{t-h(t)}^{t} \dot{x}^{T}(s) Q_{3} \dot{x}(s) d s, \\
& V_{3}(x(t), r, t) \\
& =\int_{-\bar{\tau}}^{0} \int_{t+\theta}^{t} x^{T}(s) S_{1}(r) x(s) d s d \theta \\
& +\int_{-\bar{\tau}}^{0} \int_{\theta}^{0} \int_{t+\nu}^{t} x^{T}(s) S_{2} x(s) d s d \nu d \theta, \\
& V_{4}(x(t), r, t) \\
& =\int_{-\bar{h}}^{0} \int_{t+\theta}^{t} x^{T}(s) S_{3}(r) x(s) d s d \theta \\
& +\int_{-\bar{h}}^{0} \int_{\theta}^{0} \int_{t+v}^{t} x^{T}(s) S_{4} x(s) d s d v d \theta \\
& V_{5}(x(t), r, t) \\
& =\int_{-\bar{\tau}}^{0} \int_{t+\theta}^{t} \dot{x}^{T}(s) R_{1}(r) \dot{x}(s) d s d \theta \\
& +\int_{-\bar{\tau}}^{0} \int_{\theta}^{0} \int_{t+v}^{t} \dot{x}^{T}(s) R_{2} \dot{x}(s) d s d \nu d \theta,
\end{aligned}
$$

$$
\begin{aligned}
& V_{6}(x(t), r, t) \\
& =\int_{-\bar{h}}^{0} \int_{t+\theta}^{t} \dot{x}^{T}(s) R_{3}(r) \dot{x}(s) d s d \theta \\
& \quad+\int_{-\bar{h}}^{0} \int_{\theta}^{0} \int_{t+v}^{t} \dot{x}^{T}(s) R_{4} \dot{x}(s) d s d v d \theta, \\
& V_{7}(x(t), r, t) \\
& =\int_{-\bar{\tau}}^{0} \int_{\theta}^{0} \int_{t+v}^{t} \dot{x}^{T}(s) Z_{1}(r) \dot{x}(s) d s d v d \theta \\
& \quad+\int_{-\bar{\tau}}^{0} \int_{\theta}^{0} \int_{\beta}^{0} \int_{t+v}^{t} \dot{x}^{T}(s) Z_{2} \dot{x}(s) d s d \nu d \beta d \theta, \\
& V_{8}(x(t), r, t) \\
& =\int_{-\bar{h}}^{0} \int_{\theta}^{0} \int_{t+v}^{t} \dot{x}^{T}(s) Z_{3}(r) \dot{x}(s) d s d \nu d \theta \\
& \quad+\int_{-\bar{h}}^{0} \int_{\theta}^{0} \int_{\beta}^{0} \int_{t+v}^{t} \dot{x}^{T}(s) Z_{4} \dot{x}(s) d s d v d \beta d \theta .
\end{aligned}
$$

By Definition 1 and along the trajectories of time-delay Markovian jump system (7), the weak infinitesimal operator of the stochastic process $\left.\left\{x(t), r_{t}\right\}\right|_{t \geq 0}$ is given by

$$
\begin{aligned}
& £ V_{1}(x(t), r, t)=2 x^{T}(t) P(r) \dot{x}(t)+x^{T}(t) \sum_{k=1}^{N} \pi_{r k} P(k) \\
& \cdot x(t) \\
& £ V_{2}(x(t), r, t)=x^{T}(t)\left[Q_{1}(r)+\bar{\tau} Q_{2}\right] x(t)-\left(1-\bar{\tau}_{1}\right) \\
& \text { - } x^{T}(t-\tau(t)) Q_{1}(r) x(t-\tau(t))+\dot{x}^{T}(t) Q_{3} \dot{x}(t) \\
& -\left(1-\bar{h}_{1}\right) \dot{x}^{T}(t-h(t)) Q_{3} \dot{x}(t-h(t)) \\
& +\int_{t-\tau(t)}^{t} x^{T}(s)\left[\sum_{k=1}^{N} \pi_{r k} Q_{1}(k)-Q_{2}\right] x(s) d s, \\
& £ V_{3}(x(t), r, t)=x^{T}(t)\left[\bar{\tau} S_{1}(r)+\frac{\bar{\tau}^{2}}{2} S_{2}\right] x(t) \\
& -\int_{t-\bar{\tau}}^{t} x^{T}(s) S_{1}(r) x(s) d s+\int_{-\bar{\tau}}^{0} \int_{t+\theta}^{t} x^{T}(s) \\
& \cdot\left[\sum_{k=1}^{N} \pi_{r k} S_{1}(k)-S_{2}\right] x(s) d s d \theta \\
& £ V_{4}(x(t), r, t)=x^{T}(t)\left[\bar{h} S_{3}(r)+\frac{\bar{h}^{2}}{2} S_{4}\right] x(t) \\
& -\int_{t-\bar{h}}^{t} x^{T}(s) S_{3}(r) x(s) d s+\int_{-\bar{h}}^{0} \int_{t+\theta}^{t} x^{T}(s) \\
& \cdot\left[\sum_{k=1}^{N} \pi_{r k} S_{3}(k)-S_{4}\right] x(s) d s d \theta \text {, }
\end{aligned}
$$




$$
\begin{aligned}
& £ V_{5}(x(t), r, t)=\dot{x}^{T}(t)\left[\bar{\tau} R_{1}(r)+\frac{\bar{\tau}^{2}}{2} R_{2}\right] \dot{x}(t) \\
& -\int_{t-\bar{\tau}}^{t} \dot{x}^{T}(s) R_{1}(r) \dot{x}(s) d s+\int_{-\bar{\tau}}^{0} \int_{t+\theta}^{t} \dot{x}^{T}(s) \\
& \cdot\left[\sum_{k=1}^{N} \pi_{r k} R_{1}(k)-R_{2}\right] \dot{x}(s) d s d \theta, \\
& \mathfrak{E} V_{6}(x(t), r, t)=\dot{x}^{T}(t)\left[\bar{h} R_{3}(r)+\frac{\bar{h}^{2}}{2} R_{4}\right] \dot{x}(t) \\
& -\int_{t-\bar{h}}^{t} \dot{x}^{T}(s) R_{3}(r) \dot{x}(s) d s+\int_{-\bar{h}}^{0} \int_{t+\theta}^{t} \dot{x}^{T}(s) \\
& \cdot\left[\sum_{k=1}^{N} \pi_{r k} R_{3}(k)-R_{4}\right] \dot{x}(s) d s d \theta \text {, } \\
& £ V_{7}(x(t), r, t)=\dot{x}^{T}(t)\left[\frac{\bar{\tau}^{2}}{2} Z_{1}(r)+\frac{\bar{\tau}^{3}}{6} Z_{2}\right] \dot{x}(t) \\
& -\int_{-\bar{\tau}}^{0} \int_{t+\theta}^{t} \dot{x}^{T}(s) Z_{1}(r) \dot{x}(s) d s d \theta \\
& +\int_{-\bar{\tau}}^{0} \int_{\theta}^{0} \int_{t+\beta}^{t} \dot{x}^{T}(s)\left[\sum_{k=1}^{N} \pi_{r k} Z_{1}(r)-Z_{2}\right] \dot{x}(s) d s d \beta d \theta, \\
& £ V_{8}(x(t), r, t)=\dot{x}^{T}(t)\left[\frac{\bar{h}^{2}}{2} Z_{3}(r)+\frac{\bar{h}^{3}}{6} Z_{4}\right] \dot{x}(t) \\
& -\int_{-\bar{h}}^{0} \int_{t+\theta}^{t} \dot{x}^{T}(s) Z_{3}(r) \dot{x}(s) d s d \theta \\
& +\int_{-\bar{h}}^{0} \int_{\theta}^{0} \int_{t+\beta}^{t} \dot{x}^{T}(s)\left[\sum_{k=1}^{N} \pi_{r k} Z_{3}(r)-Z_{4}\right] \dot{x}(s) d s d \beta d \theta .
\end{aligned}
$$

Applying Jensen's inequality to the integral terms in the above equations, we get

$$
\begin{aligned}
& -\int_{t-\bar{\tau}}^{t} x^{T}(s) S_{1}(r) x(s) d s \leq-\frac{1}{\bar{\tau}}\left[\int_{t-\bar{\tau}}^{t} x(s) d s\right]^{T} S_{1}(r) \\
& \cdot\left[\int_{t-\bar{\tau}}^{t} x(s) d s\right], \\
& -\int_{t-\bar{h}}^{t} x^{T}(s) S_{3}(r) x(s) d s \leq-\frac{1}{\bar{h}}\left[\int_{t-\bar{h}}^{t} x(s) d s\right]^{T} S_{3}(r) \\
& \quad \cdot\left[\int_{t-\bar{h}}^{t} x(s) d s\right], \\
& -\int_{t-\bar{\tau}}^{t} \dot{x}^{T}(s) R_{1}(r) \dot{x}(s) d s \leq-\frac{1}{\bar{\tau}}\left[x^{T}(t) R_{1}(r) x(t)\right. \\
& \quad-2 x^{T}(t-\bar{\tau}) R_{1}(r) x(t) \\
& \left.\quad+x^{T}(t-\bar{\tau}) R_{1}(r) x(t-\bar{\tau})\right]
\end{aligned}
$$

$$
\begin{aligned}
& -\int_{t-\bar{h}}^{t} \dot{x}^{T}(s) R_{3}(r) \dot{x}(s) d s \leq-\frac{1}{\bar{h}}\left[x^{T}(t) R_{3}(r) x(t)\right. \\
& -2 x^{T}(t-\bar{h}) R_{3}(r) x(t) \\
& \left.+x^{T}(t-\bar{h}) R_{3}(r) x(t-\bar{h})\right], \\
& -\int_{\bar{\tau}}^{0} \int_{t+\theta}^{t} \dot{x}^{T}(s) Z_{1}(r) \dot{x}(s) d s d \theta \leq \alpha_{1}^{T}(t) \\
& \cdot\left[\begin{array}{cc}
-Z_{1}(r)-Z_{1}^{T}(r) & \frac{1}{\bar{\tau}}\left(Z_{1}(r)+Z_{1}^{T}(r)\right) \\
* & -\frac{1}{\bar{\tau}^{2}}\left(Z_{1}(r)+Z_{1}^{T}(r)\right)
\end{array}\right] \alpha_{1}(t), \\
& -\int_{\bar{h}}^{0} \int_{t+\theta}^{t} \dot{x}^{T}(s) Z_{3}(r) \dot{x}(s) d s d \theta \leq \alpha_{2}^{T}(t) \\
& {\left[\begin{array}{cc}
-Z_{3}(r)-Z_{3}^{T}(r) & \frac{1}{\bar{h}}\left(Z_{3}(r)+Z_{3}^{T}(r)\right) \\
* & -\frac{1}{\bar{h}^{2}}\left(Z_{3}(r)+Z_{3}^{T}(r)\right)
\end{array}\right] \alpha_{2}(t),}
\end{aligned}
$$

where

$$
\begin{aligned}
& \alpha_{1}^{T}(t)=\left[\begin{array}{ll}
x^{T}(t) & \left.\int_{t-\bar{\tau}}^{t} x^{T}(s) d s\right]
\end{array}\right] \\
& \alpha_{2}^{T}(t)=\left[x^{T}(t) \int_{t-\bar{h}}^{t} x^{T}(s) d s\right] .
\end{aligned}
$$

On the other hand, for any matrices $M_{1}$ and $M_{2}$, the following equalities hold:

$$
\begin{aligned}
& 2 \gamma^{T}(t)\left[\begin{array}{c}
M_{1} \\
M_{2}
\end{array}\right]\left[\widehat{A}(r) x(t)+\widehat{A}_{d}(r) x(t-\tau(t))\right. \\
& \quad+\widehat{J}(r) \dot{x}(t-h(t))-\dot{x}(t)]=0,
\end{aligned}
$$

where $\gamma^{T}(t)=\left[x^{T}(t) \dot{x}^{T}(t)\right]$.

Combining (19) with (22), using the inequalities in (13), we can obtain

$$
£ V(x(t), r, t) \leq \zeta^{T}(t) \Phi_{i j} \zeta(t),
$$

where 


$$
\begin{aligned}
& \zeta^{T}(t)=\left[\begin{array}{lllllll}
x^{T}(t) & x^{T}(t-\tau(t)) & x^{T}(t-\bar{\tau}) & x^{T}(t-\bar{h}) & \dot{x}^{T}(t) & \dot{x}^{T}(t-h(t)) & \int_{t-\bar{\tau}}^{t} x^{T}(s) d s
\end{array} \int_{t-\bar{h}}^{t} x^{T}(s) d s\right], \\
& \Phi_{i j}=\left[\Phi_{i j}\right]_{m \times n}, \quad m, n=1,2, \ldots, 8
\end{aligned}
$$

with

$$
\begin{aligned}
& \Phi_{i j_{1,1}}=Q_{1}(r)+\bar{\tau} Q_{2}+\sum_{k=1}^{N} \pi_{r k} P(k)+\bar{\tau} S_{1}(r)+\frac{\bar{\tau}^{2}}{2} S_{2} \\
& +\bar{h} S_{3}(r)+\frac{\bar{h}^{2}}{2} S_{4}-\frac{1}{\bar{\tau}} R_{1}(r)-\frac{1}{\bar{h}} R_{3}(r) \\
& -\operatorname{sym}\left(Z_{1}(r)-Z_{3}(r)+M_{1} \widehat{A}(r)\right) \text {, } \\
& \Phi_{i j_{1,2}}=M_{1} \widehat{A}_{d}(r) \text {, } \\
& \Phi_{i j_{1,3}}=\frac{1}{\bar{\tau}} R_{1}(r), \\
& \Phi_{i j_{1,4}}=\frac{1}{\bar{h}} R_{3}(r), \\
& \Phi_{i j_{1,5}}=P(r)-M_{1}+\widehat{A}^{T}(r) M_{2}^{T} \text {, } \\
& \Phi_{i j_{1,6}}=M_{1} \widehat{J}(r) \text {, } \\
& \Phi_{i j_{1,7}}=\frac{1}{\bar{\tau}}\left(Z_{1}(r)+Z_{1}^{T}(r)\right), \\
& \Phi_{i j_{1,8}}=\frac{1}{\bar{h}}\left(Z_{3}(r)+Z_{3}^{T}(r)\right), \\
& \Phi_{i j_{2,2}}=-\left(1-\bar{\tau}_{1}\right) Q_{1}(r) \text {, } \\
& \Phi_{i j_{2,5}}=\widehat{A}_{d}^{T}(r) M_{2}^{T} \text {, } \\
& \Phi_{i j_{3,3}}=-\frac{1}{\bar{\tau}} R_{1}(r), \\
& \Phi_{i j_{4,4}}=-\frac{1}{\bar{h}} R_{3}(r) \text {, } \\
& \Phi_{i j_{5,5}}=Q_{3}+\bar{\tau} R_{1}(r)+\frac{\bar{\tau}^{2}}{2} R_{2}+\bar{h} R_{3}(r)+\frac{\bar{h}^{2}}{2} R_{4} \\
& +\frac{\bar{\tau}^{2}}{2} Z_{1}(r)+\frac{\bar{\tau}^{3}}{6} Z_{2}+\frac{\bar{h}^{2}}{2} Z_{3}(r)+\frac{\bar{h}^{3}}{6} Z_{4} \\
& -M_{2} \\
& \Phi_{i j_{5,6}}=M_{2} \widehat{J}(r) \text {, } \\
& \Phi_{i j_{6,6}}=-\left(1-\bar{h}_{1}\right) Q_{3} \text {, } \\
& \Phi_{i j_{7,7}}=-\frac{1}{\bar{\tau}} S_{1}(r)-\frac{1}{\bar{\tau}^{2}} \operatorname{sym}\left(Z_{1}(r)\right), \\
& \Phi_{i j_{8,8}}=-\frac{1}{\bar{h}} S_{3}(r)-\frac{1}{\bar{h}^{2}} \operatorname{sym}\left(Z_{3}(r)\right) \text {. }
\end{aligned}
$$

Therefore, if LMIs (13)-(15) are satisfied, (23) implies that

$$
£ V\left(x_{t}, r, t\right) \leq-\lambda\|x(t)\|^{2},
$$

where $\lambda=\lambda_{\min }\left(-\Phi_{i j}\right)$.

Now by using Dynkin's formula, we get, for any $t \geq d$,

$$
\mathbb{E} \mathfrak{E} V\left(x_{t}, r, t\right)-\mathbb{E} \mathfrak{E} V\left(x_{d}, r_{d}, d\right) \leq-\lambda \mathbb{E} \int_{d}^{t}\|x(s)\|^{2} d s,
$$

which yields $\mathbb{E} \int_{d}^{t}\|x(s)\|^{2} d s \leq \lambda^{-1} \mathbb{E} V\left(x_{d}, r_{d}, d\right)$.

Following the similar steps as in [16], it is clear that there exists a scalar $\alpha$ such that

$$
\mathbb{E} \int_{0}^{d}\|x(s)\|^{2} d s=\alpha\left\{\sup _{s \in[-d, 0]}\left\{\|\phi(s)\|^{2}\right\}\right\} .
$$

Therefore, by the definitions of $V(x(t), r, t)$ and $x(t)$, there always exists a scalar $\bar{\alpha}$ such that

$$
\begin{aligned}
\lim _{T \rightarrow \infty} \mathbb{E} \int_{0}^{T}\|x(s)\|^{2} d s & =\bar{\alpha} \mathbb{E}\left\{\sup _{s \in[-d, 0]}\left\{\|\phi(s)\|^{2}\right\}\right\} \\
& \leq M\left(r_{0}, \phi(\cdot)\right) .
\end{aligned}
$$

Considering the above condition and Definition 2, system (7) with $\omega(t) \equiv 0$ is stochastically stable. In the following, we consider the Lyapunov function (17) and the following index for system (7):

$$
\begin{gathered}
J_{T}=\mathbb{E}\left[-\langle z, Q z\rangle_{T}-2\langle z, S \omega\rangle_{T}-\langle\omega, R \omega\rangle_{T}+\delta\langle\omega,\right. \\
\left.\omega\rangle_{T}\right]=\mathbb{E}\left[\int _ { 0 } ^ { T } \left[-z(t)^{T} Q z(t)-2 z^{T}(t) S w(t)\right.\right. \\
\left.\left.-\omega^{T}(t) R \omega(t)+\delta \omega^{T}(t) \omega(t)\right] d t\right] .
\end{gathered}
$$

Under zero initial condition, it is easy to see that, for any nonzero $\omega(t) \in \mathscr{L}_{2}^{q}[0, \infty)$ and $T>0$, we have

$$
\begin{aligned}
J_{T} & \leq \mathbb{E}\left[£ V\left(x_{t}, r, t\right)+\int_{0}^{T}\left(-z(t)^{T} Q z(t)\right.\right. \\
& \left.\left.-2 z^{T}(t) S w(t)-\omega^{T}(t) R \omega(t)+\delta \omega^{T}(t) \omega(t)\right) d t\right] .
\end{aligned}
$$

Further, if conditions (13)-(15) hold for each $r \in \mathbb{M}$, then we have

$$
\begin{aligned}
& £ V\left(x_{t}, r, t\right)-z(t)^{T} Q z(t)-2 z^{T}(t) S w(t) \\
& \quad-\omega^{T}(t) R \omega(t)+\delta \omega^{T}(t) \omega(t) \leq \xi^{T}(t) \Pi_{i j} \xi(t) \\
& \quad<0,
\end{aligned}
$$


which implies from (31) that $J_{T}<0$. Thus, from the definition of $J_{T}$ in (30), we have

$$
\begin{aligned}
& \mathbb{E}\left[\int_{0}^{T}\left(z(t)^{T} \mathrm{Q} z(t)+2 z^{T}(t) S w(t)+\omega^{T}(t) \omega(t)\right) d t\right] \\
& >\delta \int_{0}^{T} \omega(t)^{T} \omega(t) d t .
\end{aligned}
$$

Therefore, for any nonzero $\omega(t) \in \mathscr{L}_{2}^{q}[0, \infty)$, the inequality (11) holds for all $T>0$. Therefore, by Definition 3, system (7) is strictly $(Q, S, R)$ dissipative.

\section{Fuzzy Controller Design}

In this section, we aim to design TS fuzzy controller for the continuous Markovian jump system (4) such that the system is stochastically stable and then strictly $(Q, S, R)$ dissipative.

Theorem 7. Consider system (4). For the given scalars $\delta>0$, $\bar{\tau}_{1}>0, \bar{h}_{1}>0$, and $\beta>0$ and matrices $Q=Q^{T}, R=R^{T}$, and $S$, there exists a feedback controller in the form of (6) such that the resulting closed-loop system of (4) is stochastically stabilized and strictly $(Q, S, R)$ dissipative if there exist matrices $\widetilde{P}(r)>0$, $\widetilde{Q}_{1}(r)>0, \widetilde{Q}_{i}>0, i=2,3, \widetilde{S}_{i}(r)>0, \widetilde{R}_{i}(r)>0, \widetilde{Z}_{i}(r)>0$, $i=1,3, \widetilde{S}_{j}>0, \widetilde{R}_{j}>0, \widetilde{Z}_{j}>0, j=2,4$, such that the following LMIs hold for each $r \in \mathbb{M}$ :

$$
\begin{aligned}
& \sum_{k=1}^{N} \pi_{r k} \widetilde{Q}_{1}(k)-\widetilde{Q}_{2}<0, \\
& \sum_{k=1}^{N} \pi_{r k} \widetilde{S}_{1}(k)-\widetilde{S}_{2}<0, \\
& \sum_{k=1}^{N} \pi_{r k} \widetilde{S}_{3}(k)-\widetilde{S}_{4}<0, \\
& \sum_{k=1}^{N} \pi_{r k} \widetilde{R}_{1}(k)-\widetilde{R}_{2}<0, \\
& \sum_{k=1}^{N} \pi_{r k} \widetilde{R}_{3}(k)-\widetilde{R}_{4}<0, \\
& \sum_{k=1}^{N} \pi_{r k} \widetilde{Z}_{1}(k)-\widetilde{Z}_{2}<0, \\
& \sum_{k=1}^{N} \pi_{r k} \widetilde{Z}_{3}(k)-\widetilde{Z}_{4}<0, \\
& \Theta_{i i}<0, \quad i=1,2, \ldots, \mathbb{S}, \\
& \Theta_{i j}+\Theta_{j i}<0, \quad i<j, i=j=1,2, \ldots, \mathbb{S},
\end{aligned}
$$

where $\Theta_{i j}=\left[\Theta_{i j}\right]_{m \times n}, m, n=1,2, \ldots, 10$, with

$$
\begin{aligned}
& \Theta_{i j_{1,1}}=\widetilde{Q}_{1}(r)+\bar{\tau} \widetilde{Q}_{2}+\sum_{k=1}^{N} \pi_{r k} \widetilde{P}(k)+\bar{\tau} \widetilde{S}_{1}(r)-\frac{\bar{\tau}^{2}}{2} \widetilde{S}_{2} \\
& +\bar{h} \widetilde{S}_{3}(r)-\frac{\bar{h}^{2}}{2} \widetilde{S}_{4}-\frac{1}{\bar{\tau}} \widetilde{R}_{1}(r)-\frac{1}{\bar{h}} \widetilde{R}_{3}(r) \\
& -\operatorname{sym}\left(\widetilde{Z}_{1}(r)\right)-\operatorname{sym}\left(\widetilde{Z}_{3}(r)\right) \\
& +\operatorname{sym}\left(A_{i}(r) X^{T}+B_{i}(r) Y_{j}(r)\right), \\
& \Theta_{i j_{1,2}}=A_{d i}(r) X^{T} \text {, } \\
& \Theta_{i j_{1,3}}=\frac{1}{\bar{\tau}} \widetilde{R}_{1}(r), \\
& \Theta_{i j_{1,4}}=\frac{1}{\bar{h}} \widetilde{R}_{3}(r), \\
& \Theta_{i j_{1,5}}=\widetilde{P}(r)-X^{T}+\beta\left(X A_{i}^{T}(r)+Y_{j}^{T}(r) B_{i}^{T}(r)\right), \\
& \Theta_{i j_{1,6}}=J_{i}(r) X^{T}, \\
& \Theta_{i j_{1,7}}=\frac{1}{\bar{\tau}}\left(\widetilde{Z}_{1}(r)+\widetilde{Z}_{1}^{T}(r)\right) \text {, } \\
& \Theta_{i j_{1,8}}=\frac{1}{\bar{h}}\left(\widetilde{Z}_{3}(r)+\widetilde{Z}_{3}^{T}(r)\right) \text {, } \\
& \Theta_{i j_{1,9}}=\widehat{B}_{1}(r)-\beta\left(X C_{i}^{T}(r)+Y_{j}^{T}(r) D_{i}^{T}(r)\right) S, \\
& \Theta_{i j_{1,10}}=\left(X C_{i}^{T}(r)+Y_{j}^{T}(r) D_{i}^{T}(r)\right) \sqrt{\bar{Q}}, \\
& \Theta_{i j_{2,2}}=-\left(1-\bar{\tau}_{1}\right) \widetilde{Q}_{1}(r) \text {, } \\
& \Theta_{i j_{2,5}}=\beta X A_{d i}^{T}, \\
& \Theta_{i j_{3,3}}=-\frac{1}{\bar{\tau}} \widetilde{R}_{1}(r), \\
& \Theta_{i j_{4,4}}=-\frac{1}{\bar{h}} \widetilde{R}_{3}(r), \\
& \Theta_{i j, 5}=\widetilde{Q}_{3}+\bar{\tau} \widetilde{R}_{1}(r)-\frac{\bar{\tau}^{2}}{2} \widetilde{R}_{2}+\bar{h} \widetilde{R}_{3}(r)-\frac{\bar{h}^{2}}{2} \widetilde{R}_{4} \\
& +\frac{\bar{\tau}^{2}}{2} \widetilde{Z}_{1}(r)+\frac{\bar{\tau}^{3}}{6} \widetilde{Z}_{2}+\frac{\bar{h}^{2}}{2} \widetilde{Z}_{3}(r)+\frac{\bar{h}^{3}}{6} \widetilde{Z}_{4} \\
& -\beta \operatorname{sym}\left(X^{T}\right) \text {, } \\
& \Theta_{i j_{5,6}}=\beta J_{i}(r) X^{T}, \\
& \Theta_{i j_{5,9}}=\beta B_{1 i} \text {, } \\
& \Theta_{i j_{6,6}}=-\left(1-\bar{h}_{1}\right) \widetilde{Q}_{3} \text {, } \\
& \Theta_{i j_{7,7}}=-\frac{1}{\bar{\tau}} \widetilde{S}_{1}(r)-\frac{1}{\bar{\tau}^{2}} \operatorname{sym}\left(\widetilde{Z}_{1}(r)+\widetilde{Z}_{1}^{T}(r)\right),
\end{aligned}
$$




$$
\begin{aligned}
\Theta_{i j_{8,8}} & =-\frac{1}{\bar{h}} \widetilde{S}_{3}(r)-\frac{1}{\bar{h}^{2}}\left(\widetilde{Z}_{3}(r)+\widetilde{Z}_{3}^{T}(r)\right), \\
\Theta_{i j_{9,9}} & =-(R-\delta I), \\
\Theta_{i j_{10,10}} & =-I
\end{aligned}
$$

and the remaining parameters are zero. Moreover, the desired state feedback controller gain can be obtained as $K_{j}=$ $Y_{j}(r) X^{-1^{T}}$.

Proof. In order to obtain the feedback controller gain matrices, take $W=\{X, X, \ldots, X\} \in \mathbb{R}^{8 \times 8}$. Before and after multiplying (13)-(15) by $\operatorname{diag}\{W, I\}$ and its transpose, respectively, where $X=M_{1}^{-1}$, and letting $\widetilde{P}(r)=X P(r) X^{T}, \widetilde{Q}_{1 r}=$ $X Q_{1}(r) X^{T}, \widetilde{Q}_{i}=X Q_{i} X^{T}, i=2,3, \widetilde{S}_{i}(r)=X S_{i}(r) X^{T}$, $\widetilde{R}_{i}(r)=X R_{i}(r) X^{T}, \widetilde{Z}_{i}(r)=X Z_{i}(r) X^{T}, i=1,3, \widetilde{S}_{j}=$ $X S_{j} X^{T}, \widetilde{R}_{j}=X R_{j} X^{T}, \widetilde{Z}_{j}=X Z_{j} X^{T}, j=2,4$, the matrix $M_{2}=\beta M_{1}$, where $\beta$ is the designing parameter and $Y_{j}(r)=$ $K_{j}(r) X^{T}$, we can obtain the LMIs (34). Hence, system (4) is stochastically stabilized and strictly $(Q, S, R)$ dissipative through the proposed stabilized feedback controller. The proof is completed.

In the following corollary, we will consider the dissipative controller design for fuzzy neutral Markov jump system with constant delays. The time delays $\tau(t)$ and $h(t)$ are constant, which can be described as $\tau(t)=\tau_{1}>0, h(t)=h_{1}>0$. Then the fuzzy Markov jump system (4) can be written as the following fuzzy model.

Plant Rule $i$. If $\mu_{1}(t)$ is $F_{1}^{i}, \mu_{2}(t)$ is $F_{2}^{i}$, and $\ldots, \mu_{g}(t)$ is $F_{g}^{i}$, then

$$
\begin{aligned}
\dot{x}(t)= & A_{i}(r) x(t)+A_{d i}(r) x\left(t-\tau_{1}\right) \\
& +J_{i}(r) \dot{x}\left(t-h_{1}\right)+B_{i}(r) u(t) \\
& +B_{1 i}(r) \omega(t), \\
z(t)= & C_{i}(r) x(t)+D_{i}(r) u(t), \\
x(t)= & \phi(t), \\
r= & r_{0}, \quad t \in[-d, 0], i=1,2, \ldots, \mathbb{S} .
\end{aligned}
$$

Corollary 8. For the given scalars $\delta>0, \tau_{1}>0, h_{1}>0$, $\beta>0$ and matrices $Q=Q^{T}, R=R^{T}, S$, system (36) is stochastically stabilized through the controller (6) and strictly $(Q, S, R)$ dissipative, if there exist matrices $\widetilde{P}(r)>0, \widetilde{Q}_{1}(r)>0$, $\widetilde{Q}_{i}>0, i=2,3, \widetilde{S}_{i}(r)>0, \widetilde{R}_{i}(r)>0, \widetilde{Z}_{i}(r)>0, i=1,3, \widetilde{S}_{j}>0$, $\widetilde{R}_{j}>0, \widetilde{Z}_{j}>0, j=2,4$, such that the following LMIs hold for each $r \in \mathbb{M}$ :

$$
\begin{gathered}
\sum_{k=1}^{N} \pi_{r k} \widetilde{Q}_{1}(k)-\widetilde{Q}_{2}<0, \\
\sum_{k=1}^{N} \pi_{r k} \widetilde{S}_{1}(k)-\widetilde{S}_{2}<0,
\end{gathered}
$$

$$
\begin{aligned}
& \sum_{k=1}^{N} \pi_{r k} \widetilde{S}_{3}(k)-\widetilde{S}_{4}<0, \\
& \sum_{k=1}^{N} \pi_{r k} \widetilde{R}_{1}(k)-\widetilde{R}_{2}<0, \\
& \sum_{k=1}^{N} \pi_{r k} \widetilde{R}_{3}(k)-\widetilde{R}_{4}<0, \\
& \sum_{k=1}^{N} \pi_{r k} \widetilde{Z}_{1}(k)-\widetilde{Z}_{2}<0, \\
& \sum_{k=1}^{N} \pi_{r k} \widetilde{Z}_{3}(k)-\widetilde{Z}_{4}<0, \\
& \theta_{i i}<0, \quad i=1,2, \ldots, \mathbb{S}, \\
& \theta_{i j}+\theta_{j i}<0, \quad i<j, i=j=1,2, \ldots, \mathbb{S},
\end{aligned}
$$

where $\theta_{i j}=\left[\theta_{i j}\right]_{m \times n}, m, n=1,2, \ldots, 10$, with

$$
\begin{aligned}
& \theta_{i j_{1,1}}=\widetilde{Q}_{1}(r)+\tau_{1} \widetilde{Q}_{2}+\sum_{k=1}^{N} \pi_{r k} \widetilde{P}(k)+\tau_{1} \widetilde{S}_{1}(r)-\frac{\tau_{1}^{2}}{2} \widetilde{S}_{2} \\
& +h_{1} \widetilde{S}_{3}(r)-\frac{h_{1}^{2}}{2} \widetilde{S}_{4}-\frac{1}{\tau_{1}} \widetilde{R}_{1}(r)-\frac{1}{h_{1}} \widetilde{R}_{3}(r) \\
& -\operatorname{sym}\left(\widetilde{Z}_{1}(r)\right)-\operatorname{sym}\left(\widetilde{Z}_{3}(r)\right) \\
& +\operatorname{sym}\left(A_{i}(r) X^{T}+B_{i}(r) Y_{j}(r)\right) \text {, } \\
& \theta_{i j_{1,2}}=A_{d i}(r) X^{T} \\
& \theta_{i j_{1,3}}=\frac{1}{\tau_{1}} \widetilde{R}_{1}(r) \text {, } \\
& \theta_{i j 1,4}=\frac{1}{h_{1}} \widetilde{R}_{3}(r) \text {, } \\
& \theta_{i j_{1,5}}=\widetilde{P}(r)-X^{T}+\beta\left(X A_{i}^{T}(r)+Y_{j}^{T}(r) B_{i}^{T}(r)\right) \text {, } \\
& \theta_{i j_{1,6}}=J_{i}(r) X^{T} \text {, } \\
& \theta_{i j_{1,7}}=\frac{1}{\tau_{1}}\left(\widetilde{Z}_{1}(r)+\widetilde{Z}_{1}^{T}(r)\right) \text {, } \\
& \theta_{i j_{1,8}}=\frac{1}{h_{1}}\left(\widetilde{Z}_{3}(r)+\widetilde{Z}_{3}^{T}(r)\right) \text {, } \\
& \theta_{i j_{1,9}}=B_{1 i}(r)-\beta\left(X C_{i}^{T}(r)+Y_{j}^{T}(r) D_{i}^{T}(r)\right) S \text {, } \\
& \theta_{i j_{1,10}}=\left(X C_{i}^{T}(r)+Y_{j}^{T}(r) D_{i}^{T}(r)\right) \sqrt{\bar{Q}}, \\
& \theta_{i j_{2,2}}=-\widetilde{Q}_{1}(r) \text {, }
\end{aligned}
$$




$$
\begin{aligned}
& \theta_{i j_{2,5}}=\beta X A_{d i}^{T}(r) \\
& \theta_{i j_{3,3}}=-\frac{1}{\tau_{1}} \widetilde{R}_{1}(r), \\
& \theta_{i j_{4,4}}=-\frac{1}{h_{1}} \widetilde{R}_{3}(r) \\
& \theta_{i j_{5,5}}=\widetilde{Q}_{3}+\tau_{1} \widetilde{R}_{1}(r)-\frac{\tau_{1}^{2}}{2} \widetilde{R}_{2}+h_{1} \widetilde{R}_{3}(r)-\frac{h_{1}^{2}}{2} \widetilde{R}_{4} \\
& +\frac{\tau_{1}^{2}}{2} \widetilde{Z}_{1}(r)+\frac{\tau_{1}^{3}}{6} \widetilde{Z}_{2}+\frac{h_{1}^{2}}{2} \widetilde{Z}_{3}(r)+\frac{h_{1}^{3}}{6} \widetilde{Z}_{4} \\
& -\beta \operatorname{sym}\left(X^{T}\right) \text {, } \\
& \theta_{i j_{5,6}}=\beta J_{i}(r) X^{T} \text {, } \\
& \theta_{i j_{5,9}}=\beta B_{1 i}(r) \text {, } \\
& \theta_{i j_{6,6}}=-\widetilde{Q}_{3} \text {, } \\
& \theta_{i j_{7,7}}=-\frac{1}{\tau_{1}} \widetilde{S}_{1}(r)-\frac{1}{\tau_{1}^{2}} \operatorname{sym}\left(\widetilde{Z}_{1}(r)+\widetilde{Z}_{1}^{T}(r)\right), \\
& \theta_{i j_{8,8}}=-\frac{1}{h_{1}} \widetilde{S}_{3}(r)-\frac{1}{h_{1}^{2}} \operatorname{sym}\left(\widetilde{Z}_{3}(r)+\widetilde{Z}_{3}^{T}(r)\right) \text {, } \\
& \theta_{i j_{9,9}}=-(R-\delta I) \text {, } \\
& \theta_{i j_{10,10}}=-I
\end{aligned}
$$

and the remaining parameters are zero. In this case, the desired state feedback controller gain can be given as $K_{j}=Y_{j}(r) X^{-1^{T}}$.

Proof. Consider the same LKF as in the Theorem 6, $V_{i}(x(t) r, t)=\sum_{i=1}^{8} V_{i}(x(t), r, t)$, in which $V_{2}(x(t), r, t)$ is replaced with $V_{2}(x(t), r, t)=\int_{t-\bar{\tau}}^{t} x^{T}(s) Q_{1}(r) x(s) d s+$ $\int_{-\bar{\tau}}^{0} \int_{t+\theta}^{t} x^{T}(s) Q_{2} x(s) d s d \theta+\int_{t-\bar{h}}^{t} \dot{x}^{T}(s) Q_{3} \dot{x}(s) d s$ and the remaining terms of LKF are the same as in Theorem 6. By following the similar steps as in Theorem 7 with some modifications, we can obtain the desired result. The proof is completed.

\section{Numerical Example}

Example 9. In this section, we present a numerical example with simulation to illustrate effectiveness and applicability of the proposed dissipative control law. Consider the trucktrailer model which is borrowed from [39] described by the following dynamical system with two modes:

$$
\begin{gathered}
\dot{x}_{1}(t)=-a(r) \frac{v \bar{t}}{L t_{0}} x_{1}(t)-(1-a(r)) \frac{v \bar{t}}{L t_{0}} x_{1}(t-\tau(t)) \\
-(1-b(r)) \dot{x}_{1}(t-h(t))+\frac{v \bar{t}}{l t_{0}} u(t)+\frac{v \bar{t}}{L t_{0}} \omega(t),
\end{gathered}
$$

$$
\begin{gathered}
\dot{x}_{2}(t)=a(r) \frac{v \bar{t}}{L t_{0}} x_{1}(t)+(1-a(r)) \frac{v \bar{t}}{L t_{0}} x_{1}(t-\tau(t)) \\
-(1-b(r)) \dot{x}_{2}(t-h(t)), \\
\dot{x}_{3}(t)=\frac{v \bar{t}}{t_{0}} \sin \left[x_{2}(t)+a(r) \frac{v \bar{t}}{2 L} x_{1}(t)\right. \\
\left.+(1-a(r)) \frac{v \bar{t}}{L t_{0}} x_{1}(t-\tau(t))\right]-(1-b(r)) \dot{x}_{3}(t \\
-h(t)), \\
z(t)=-a(r) \frac{v \bar{t}}{L t_{0}} x_{1}(t)+\frac{v \bar{t}}{l t_{0}} u(t),
\end{gathered}
$$

where $x_{1}(t)$ is the angle difference between truck and trailer; $x_{2}(t)$ is the angle of trailer; $x_{3}(t)$ is the vertical position of rear of trailer; $u(t)$ is the steering angle; $\omega(t)$ is the disturbance; and $z(t)$ is the output angle variable. In order to verify the results, we borrow the model parameters from [39] such as $l=$ 2.8, $L=5.5, v=-1.0, \bar{t}=2.0$, and $t_{0}=0.5$. Also $a(r)$ and $b(r)$ are jumping parameters with values $a(1)=0.7, a(2)=0.5$, $b(1)=0.5$, and $b(2)=0.3$. Further, the transition probability matrix that relates two operation modes is taken as

$$
\Pi=\left[\begin{array}{cc}
-2.0 & 2.0 \\
0.6 & -0.6
\end{array}\right] \text {. }
$$

Let $\mu(t)=x_{2}(t)+a(r)(v \bar{t} / 2 L) x_{1}(t)+(1-a(r))\left(v \bar{t} / L t_{0}\right) x_{1}(t-$ $\tau(t))$. Under the condition $-179.4270^{\circ}<\mu(t)<179.4270^{\circ}$, the nonlinear term $\sin (\mu(t))$ can be exactly represented as $\sin (\mu(t))=h_{1}(\mu(t)) \mu(t)+h_{2}(\mu(t)) \bar{\omega} \mu(t)$ with $\bar{\omega}=10^{-2} / \pi$, where $h_{1}(\mu(t)), h_{2}(\mu(t)) \in[0,1]$ such that $h_{1}(\mu(t))+$ $h_{2}(\mu(t))=1$. By solving the equations, the membership functions $h_{1}(\mu(t))$ and $h_{2}(\mu(t))$ are obtained as [41]

$$
\begin{aligned}
& h_{1}(\mu(t))= \begin{cases}\frac{\sin (\mu(t))-\bar{\omega} \mu(t)}{\mu(t) \cdot(1-\bar{\omega})}, & \mu(t) \neq 0, \\
1, & \mu(t)=0,\end{cases} \\
& h_{2}(\mu(t))= \begin{cases}\frac{\mu(t)-\sin (\mu(t))}{\mu(t) \cdot(1-\bar{\omega})}, & \mu(t) \neq 0, \\
0 & \mu(t)=0 .\end{cases}
\end{aligned}
$$

Further, we use the following fuzzy T-S models to approximate the neutral Markovian jump system.

Subsystem 1. If $\mu(t)$ is about $0(\mathrm{rad})$, then

$$
\begin{aligned}
\dot{x}(t)= & A_{1}(r) x(t)+A_{d 1}(r) x(t-\tau(t)) \\
& +J_{1}(r) \dot{x}(t-h(t))+B_{1}(r) u(t) \\
& +B_{11}(r) \omega(t), \\
z(t)= & C_{1}(r) x(t)+D_{1}(r) u(t) .
\end{aligned}
$$


Subsystem 2. If $\mu(t)$ is about $\pi(\mathrm{rad})$ and $-\pi(\mathrm{rad})$, then

$$
\begin{aligned}
\dot{x}(t)= & A_{2}(r) x(t)+A_{d 2}(r) x(t-\tau(t)) \\
& +J_{2}(r) \dot{x}(t-h(t))+B_{2}(r) u(t) \\
& +B_{12}(r) \omega(t), \\
z(t)= & C_{2}(r) x(t)+D_{2}(r) u(t),
\end{aligned}
$$

where $x(t)=\left[\begin{array}{lll}x_{1}^{T}(t) & x_{2}^{T}(t) x_{3}^{T}(t)\end{array}\right]^{T}$ and

$$
\begin{aligned}
& A_{1}(r)=\left[\begin{array}{ccc}
-a(r) \frac{v \bar{t}}{L t_{0}} & 0 & 0 \\
a(r) \frac{v \bar{t}}{L t_{0}} & 0 & 0 \\
a(r) \frac{v^{2} \bar{t}^{2}}{2 L t_{0}} & \frac{v \bar{t}}{t_{0}} & 0
\end{array}\right], \\
& A_{d 1}(r)=\left[\begin{array}{ccc}
-(1-a(r)) \frac{v \bar{t}}{L t_{0}} & 0 & 0 \\
(1-a(r)) \frac{v \bar{t}}{L t_{0}} & 0 & 0 \\
(1-a(r)) \frac{v^{2} \bar{t}^{2}}{2 L t_{0}} & 0 & 0
\end{array}\right] \text {, } \\
& A_{2}(r)=\left[\begin{array}{ccc}
-a(r) \frac{v \bar{t}}{L t_{0}} & 0 & 0 \\
a(r) \frac{v \bar{t}}{L t_{0}} & 0 & 0 \\
a(r) \frac{\bar{\omega} v^{2} \bar{t}^{2}}{2 L t_{0}} & \frac{d v \bar{t}}{t_{0}} & 0
\end{array}\right] \\
& A_{d 2}(r)=\left[\begin{array}{ccc}
-(1-a(r)) \frac{v \bar{t}}{L t_{0}} & 0 & 0 \\
(1-a(r)) \frac{v \bar{t}}{L t_{0}} & 0 & 0 \\
(1-a(r)) \frac{\bar{\omega} v^{2} \bar{t}^{2}}{2 L t_{0}} & 0 & 0
\end{array}\right] \text {, } \\
& B_{1}(r)=B_{2}(r)=\left[\begin{array}{lll}
\frac{\bar{t}}{l t_{0}} & 0 & 0
\end{array}\right]^{T} \text {, } \\
& J_{1}(r)=J_{2}(r) \\
& =\left[\begin{array}{ccc}
(1-b(r)) & 0 & 0 \\
0 & (1-b(r)) & 0 \\
0 & 0 & (1-b(r))
\end{array}\right] \text {, } \\
& C_{1}(r)=C_{2}(r)=\left[\begin{array}{lll}
-0.7273 & 0 & 0
\end{array}\right], \\
& D_{1}(r)=D_{2}(r)=-1.4286 \text {, } \\
& B_{11}(r)=B_{12}(r)=\left[\begin{array}{lll}
-0.7273 & 0 & 0
\end{array}\right]^{T} \text {. }
\end{aligned}
$$

TABLE 1: Calculated minimum $\gamma$ for various values of $\beta$.

\begin{tabular}{lcccccc}
\hline & $\beta$ & 0.8 & 1.0 & 1.2 & 1.4 & 1.6 \\
\hline$H_{\infty}$ case & $\gamma_{\min }$ & 1.6980 & 1.7986 & 1.8864 & 1.9637 & 2.0329 \\
\hline Passivity case & $\gamma_{\min }$ & 0.7785 & 0.8726 & 0.9544 & 1.0264 & 1.0902 \\
\hline $\begin{array}{l}\text { Mixed } H_{\infty} \text { and } \\
\text { passivity case } \\
\text { when } \theta=0.5\end{array}$ & $\gamma_{\min }$ & 1.0880 & 1.1678 & 1.2384 & 1.3009 & 1.3571 \\
\hline
\end{tabular}

For the given values $\bar{\tau}=0.2, \bar{h}=0.1, \bar{\tau}_{1}=0.1$, and $\bar{h}_{1}=0.2$, by solving the LMIs in Theorem 7, using MATLAB LMI tool box, we can get feasible solution which is not given here due to the page constraint. The calculated minimum allowable $\gamma$ for different values of the designing parameter $\beta$ is given in Table 1. Our purpose is to design the state feedback dissipative controller gain in (6) such that system (39) is stochastically stable and strictly dissipative. Also, with the use of Theorem 7 and based on Remark 4, the obtained $H_{\infty}$ controller, passivity controller, mixed $H_{\infty}$, and passivity controller gain matrices can be calculated as follows. For instance, if we choose the matrices $Q=-0.25, R=5, S=0.9$, and $\beta=1.6$, by solving the LMIs in Theorem 7 with the help of the MATLAB LMI tool box, we can get feasible solution which is not given here due to the page constraint. Moreover, the corresponding gain matrices for the dissipative case are obtained as

$$
\begin{aligned}
& K_{1}(1)=\left[\begin{array}{lll}
0.8000 & -0.3326 & 0.0087
\end{array}\right], \\
& K_{1}(2)=\left[\begin{array}{lll}
3.3432 & -1.1309 & 0.0142
\end{array}\right], \\
& K_{2}(1)=\left[\begin{array}{lll}
0.9171 & -0.3318 & 0.0085
\end{array}\right], \\
& K_{2}(2)=\left[\begin{array}{lll}
3.3566 & -1.0987 & 0.0134
\end{array}\right] .
\end{aligned}
$$

Further, when $\beta=1.6$, the corresponding gain matrices for $H_{\infty}$ based control case are obtained as

$$
\begin{aligned}
& K_{1}(1)=\left[\begin{array}{lll}
1.6068 & -0.2837 & 0.0059
\end{array}\right], \\
& K_{1}(2)=\left[\begin{array}{lll}
3.7634 & -0.6696 & 0.0108
\end{array}\right], \\
& K_{2}(1)=\left[\begin{array}{lll}
1.9174 & -0.2351 & 0.0075
\end{array}\right], \\
& K_{2}(2)=\left[\begin{array}{lll}
3.7535 & -0.6257 & 0.0103
\end{array}\right]
\end{aligned}
$$

with a minimum $H_{\infty}$ performance index given by $\gamma_{\min }=$ 2.0329. Similarly, when $\beta=1.6$, that is, for the passivity based control case, the corresponding gain matrices can be calculated as

$$
\begin{aligned}
& K_{1}(1)=\left[\begin{array}{lll}
5.5399 & -11.8326 & 0.1936
\end{array}\right], \\
& K_{1}(2)=\left[\begin{array}{lll}
12.0810 & -26.7460 & 0.3402
\end{array}\right], \\
& K_{2}(1)=\left[\begin{array}{lll}
6.1193 & -11.6389 & 0.1683
\end{array}\right], \\
& K_{2}(2)=\left[\begin{array}{lll}
11.7724 & -24.8721 & 0.31687
\end{array}\right]
\end{aligned}
$$

with a minimum performance index given by $\gamma_{\min }=1.0902$. Finally, when $\beta=1.6$, that is, for the mixed $H_{\infty}$ and passivity 

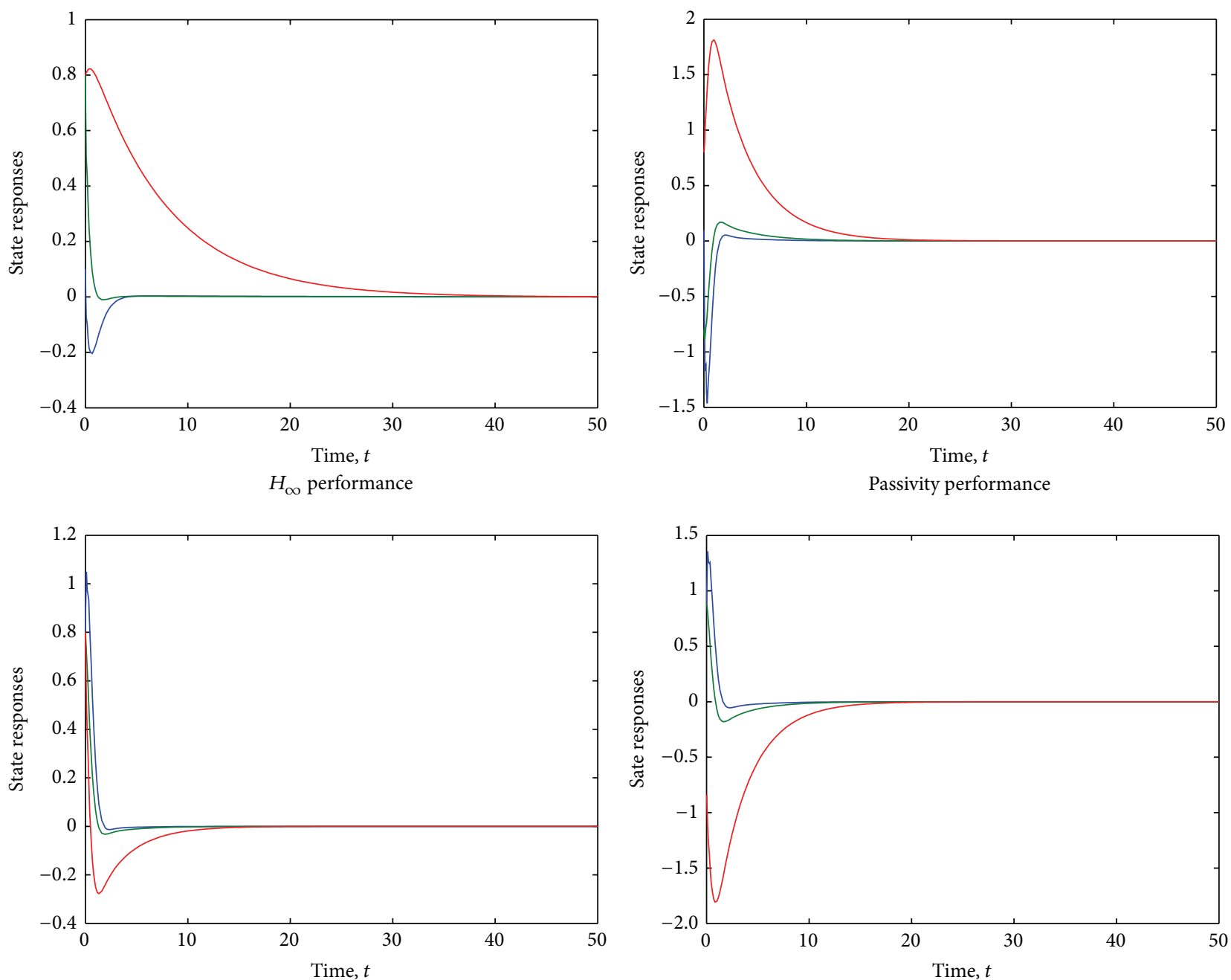

Mixed $H_{\infty}$ and passivity performance

Dissipativity performance
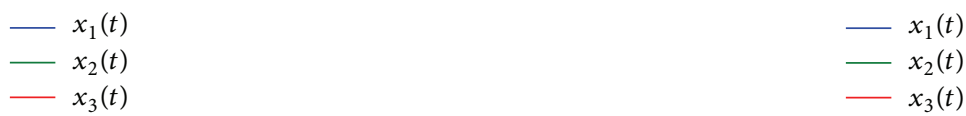

FIGURE 1: State trajectories of the system.

performance based control case, the gain matrices can be obtained as

$$
\begin{aligned}
& K_{1}(1)=\left[\begin{array}{lll}
5.7999 & -10.6849 & 0.1525
\end{array}\right] \\
& K_{1}(2)=\left[\begin{array}{lll}
11.5721 & -22.3547 & 0.2677
\end{array}\right] \\
& K_{2}(1)=\left[\begin{array}{lll}
6.1304 & -10.2237 & 0.1389
\end{array}\right] \\
& K_{2}(2)=\left[\begin{array}{lll}
11.2773 & -20.9786 & 0.2531
\end{array}\right]
\end{aligned}
$$

with a minimum performance index given by $\gamma_{\min }=1.3571$.

Further, to show the effectiveness of the proposed controller, simulation results are presented in Figures 1 and 2. For the simulation purpose, the disturbance input is assumed as $\omega(t)=0.5 \sin (t)$ and the initial condition is taken as $x(0)=\left[\begin{array}{lll}0.8 & 0.8 & 0.8\end{array}\right]^{T}$. Simulation results for state responses of the fuzzy system for $H_{\infty}$, passivity, mixed $H_{\infty}$ and passivity, and dissipativity performances are provided in Figure 1. Moreover, Figure 2 represents the trajectories of the dissipative control law and output responses of the systems.

It should be mentioned that the obtained results can also be applied to design $H_{\infty}$, passivity, and mixed $H_{\infty}$ and passivity control for TS fuzzy model Markovian jump neutral systems with time delays. From the obtained results, it is concluded that these different control processes can be achieved for the truck-trailer model with the same design process, so it can potentially save time and cost in the controller design.

Remark 10. It should be noted that the dissipative control formulation is general and flexible. By choosing different values of $Q, S$, and $R$, the dissipative control can include various types of control as special cases. Also, the simulation result reveals that it is possible to obtain the controller 

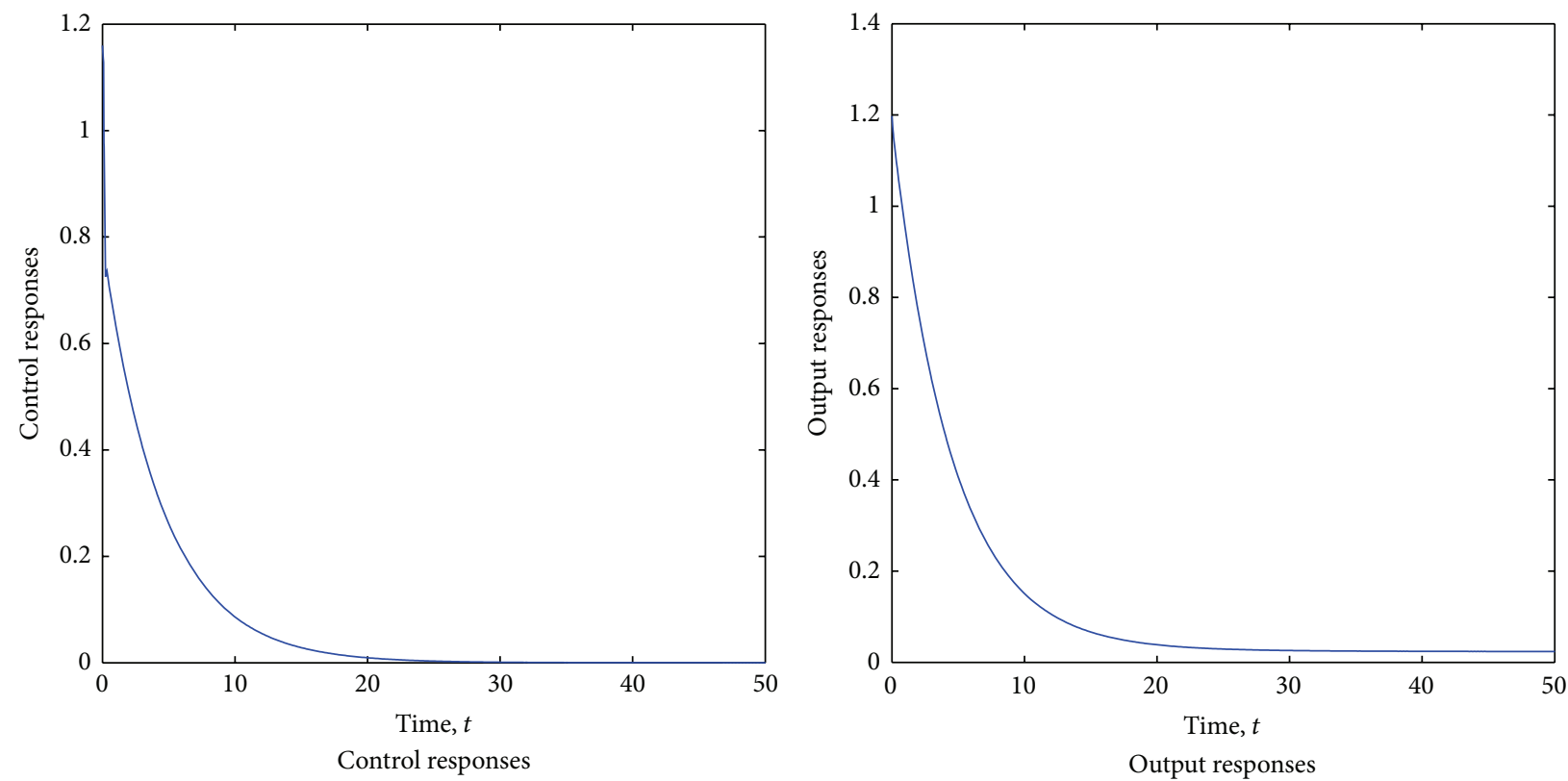

FIgURE 2: Dissipative performance of the system.

with minimum control effort from the proposed dissipative controller law to achieve the desired performance.

\section{Conclusion}

This paper focuses on the robust dissipative control problem for a class of TS fuzzy neutral Markovian jump systems. In particular, as special cases, the design methods of $H_{\infty}$ and passive controller can also be obtained. This explains the fact that the dissipative control ia a unified framework for $H_{\infty}$ and passivity control. It is shown that a desired state feedback dissipative control can be constructed when the given LMIs are feasible. By employing the Lyapunov approach, some sufficient conditions are derived in terms of linear matrix inequalities which ensures that the closed-loop system is strictly dissipative and stochastically stable. Finally, a numerical example based on truck-trailer model with simulation result is provided to illustrate the effectiveness and validity of the obtained design technique. The dissipative analysis and synthesis of control to uncertain interval type 2 fuzzy systems with Markovian jumps via delta operator approach will be the topic of our future research.

\section{Conflict of Interests}

The authors declare that there is no conflict of interests regarding the publication of this paper.

\section{Acknowledgment}

This work was supported by the National Research Foundation of Korea (NRF) grant funded by the Brain Korea 21 PLUS (no. 21A20131400002).

\section{References}

[1] H. Li, Y. Gao, L. Wu, and H. K. Lam, "Fault detection for T$S$ fuzzy time-delay systems: delta operator and input-output methods," IEEE Transactions on Cybernetics, vol. 45, pp. 229241, 2015

[2] O. M. Kwon, M. J. Park, S. M. Lee, and J. H. Park, "Augmented Lyapunov-Krasovskii functional approaches to robust stability criteria for uncertain Takagi-Sugeno fuzzy systems with timevarying delays," Fuzzy Sets and Systems, vol. 201, pp. 1-19, 2012.

[3] L. Wu, X. Su, P. Shi, and J. Qiu, "Model approximation for discrete-time state-delay systems in the T-S fuzzy framework," IEEE Transactions on Fuzzy Systems, vol. 19, no. 2, pp. 366-378, 2011.

[4] W. Assawinchaichote, "Further results on robust fuzzy dynamic systems with LIM D-stability constraints," International Journal of Applied Mathematics and Computer Science, vol. 24, pp. 785794, 2014.

[5] H. Li, H. Liu, H. Gao, and P. Shi, "Reliable fuzzy control for active suspension systems with actuator delay and fault," IEEE Transactions on Fuzzy Systems, vol. 20, no. 2, pp. 342-357, 2012.

[6] Z. G. Wu, P. Shi, H. Su, and J. Chu, "Reliable $H_{\infty}$ control for discrete-time fuzzy systems with infinite distributed delay," IEEE Transactions on Fuzzy Systems, vol. 20, no. 1, pp. 22-31, 2012.

[7] S. Zhang, Z. Wang, D. Ding, and H. Shu, " $H_{\infty}$ fuzzy control with randomly occurring infinite distributed delays and channel fadings," IEEE Transactions on Fuzzy Systems, vol. 22, no. 1, pp. 189-200, 2014.

[8] R. Sakthivel, P. Vadivel, K. Mathiyalagan, and A. Arunkumar, "Fault-distribution dependent reliable $H_{\infty}$ control for TakagiSugeno fuzzy systems," Journal of Dynamic Systems, Measurement and Control, vol. 136, no. 2, Article ID 021021, 11 pages, 2014.

[9] R. Sakthivel, P. Vadivel, K. Mathiyalagan, and J. H. Park, "Robust control for fuzzy nonlinear uncertain systems with discrete and 
distributed time delays," Zeitschrift für Naturforschung A, vol. 69, pp. 569-580, 2014.

[10] L. Sheng and X. Ma, "Stability analysis and controller design of interval type-2 fuzzy systems with time delay," International Journal of Systems Science, vol. 45, no. 5, pp. 977-993, 2014.

[11] X. Su, P. Shi, L. Wu, and Y.-D. Song, "A novel approach to filter design for T-S fuzzy discrete-time systems with time-varying delay," IEEE Transactions on Fuzzy Systems, vol. 20, no. 6, pp. 1114-1129, 2012.

[12] H. Li, H. Gao, P. Shi, and X. Zhao, "Fault-tolerant control of Markovian jump stochastic systems via the augmented sliding mode observer approach," Automatica, vol. 50, no. 7, pp. 18251834, 2014.

[13] Q. Zhu and J. Cao, "Robust exponential stability of markovian jump impulsive stochastic Cohen-Grossberg neural networks with mixed time delays," IEEE Transactions on Neural Networks, vol. 21, no. 8, pp. 1314-1325, 2010.

[14] Q. Zhu and J. Cao, "Stability analysis of markovian jump stochastic BAM neural networks with impulse control and mixed time delays," IEEE Transactions on Neural Networks and Learning Systems, vol. 23, no. 3, pp. 467-479, 2012.

[15] L. Wu, X. Su, and P. Shi, "Sliding mode control with bounded L2 gain performance of Markovian jump singular time-delay systems," Automatica, vol. 48, no. 8, pp. 1929-1933, 2012.

[16] Z. Wu, H. Su, and J. Chu, " $H_{\infty}$ filtering for singular Markovian jump systems with time delay," International Journal of Robust and Nonlinear Control, vol. 20, no. 8, pp. 939-957, 2010.

[17] Q. Zhu and J. Cao, "Exponential stability of stochastic neural networks with both Markovian jump parameters and mixed time delays," IEEE Transactions on Systems, Man, and Cybernetics, Part B: Cybernetics, vol. 41, no. 2, pp. 341-353, 2011.

[18] P. Balasubramaniam, V. M. Revathi, and J. H. Park, " $L_{2}-L_{\infty}$ filtering for neutral Markovian switching system with modedependent time-varying delays and partially unknown transition probabilities," Applied Mathematics and Computation, vol. 219, no. 17, pp. 9524-9542, 2013.

[19] Q. Zhu and J. Cao, "Stability of Markovian jump neural networks with impulse control and time varying delays," Nonlinear Analysis. Real World Applications, vol. 13, no. 5, pp. 2259-2270, 2012.

[20] Q. Zhu, "pth moment exponential stability of impulsive stochastic functional differential equations with Markovian switching," Journal of the Franklin Institute, vol. 351, no. 7, pp. 3965-3986, 2014.

[21] J.-W. Wang, H.-N. Wu, L. Guo, and Y.-S. Luo, "Robust $H_{\infty}$ fuzzy control for uncertain nonlinear Markovian jump systems with time-varying delay," Fuzzy Sets and Systems, vol. 212, pp. 41-61, 2013.

[22] M. Chadli, H. R. Karimi, and P. Shi, "On stability and stabilization of singular uncertain Takagi-Sugeno fuzzy systems," Journal of the Franklin Institute, vol. 351, no. 3, pp. 1453-1463, 2014.

[23] D. W. Kim and H. J. Lee, "Sampled-data observer-based output-feedback fuzzy stabilization of nonlinear systems: exact discrete-time design approach," Fuzzy Sets and Systems, vol. 201, pp. 20-39, 2012.

[24] H. Li, X. Jing, H. K. Lam, and P. Shi, "Fuzzy sampleddata control for uncertain vehicle suspension systems," IEEE Transactions on Cybernetics, vol. 44, no. 7, pp. 1111-1126, 2014.

[25] H. K. Lam, "Stabilization of nonlinear systems using sampleddata output-feedback fuzzy controller based on polynomialfuzzy-model-based control approach," IEEE Transactions on
Systems, Man, and Cybernetics, Part B: Cybernetics, vol. 42, no. 1, pp. 258-267, 2012.

[26] K. Mathiyalagan, R. Sakthivel, and S. M. Anthoni, "New stability criteria for stochastic Takagi-Sugeno fuzzy systems with timevarying delays," Journal of Dynamic Systems, Measurement and Control, vol. 136, no. 2, Article ID 021013, 2014.

[27] Q. Gao, G. Feng, Z. Xi, Y. Wang, and J. Qiu, "A new design of robust $H_{\infty}$ sliding mode control for uncertain stochastic TS fuzzy time-delay systems," IEEE Transactions on Cybernetics, vol. 44, pp. 1556-1566, 2014.

[28] Y. Zhang, P. Shi, S. K. Nguang, H. R. Karimi, and R. K. Agarwal, "Robust finite-time fuzzy $H_{\infty}$ control for uncertain time-delay systems with stochastic jumps," Journal of the Franklin Institute, vol. 351, no. 8, pp. 4211-4229, 2014.

[29] C. Peng and Q.-L. Han, "Delay-range-dependent robust stabilization for uncertain T-S fuzzy control systems with interval time-varying delays," Information Sciences, vol. 181, no. 19, pp. 4287-4299, 2011.

[30] L. Li, Q. Zhang, and B. Zhu, " $H_{\infty}$ fuzzy control for nonlinear time-delay singular Markovian jump systems with partly unknown transition rates," Fuzzy Sets and Systems, vol. 254, pp. 106-125, 2014.

[31] D. J. Hill and P. J. Moylan, "Dissipative dynamical systems: basic input-output and state properties," Journal of the Franklin Institute, vol. 309, no. 5, pp. 327-357, 1980.

[32] J. C. Willems, "Dissipative dynamical systems. I. General theory," Archive for Rational Mechanics and Analysis, vol. 45, pp. 321-351, 1972.

[33] Z. Guo, J. Wang, and Z. Yan, "Global exponential dissipativity and stabilization of memristor-based recurrent neural networks with time-varying delays," Neural Networks, vol. 48, pp. 158-172, 2013.

[34] L. Sheng, M. Gao, and W. Zhang, "Dissipative control for Markov jump non-linear stochastic systems based on T-S fuzzy model," International Journal of Systems Science, vol. 45, no. 5, pp. 1213-1224, 2014.

[35] Z. Feng and J. Lam, "Reliable dissipative control for singular Markovian systems," Asian Journal of Control, vol. 15, no. 3, pp. 901-910, 2013.

[36] L. Wu, X. Yang, and H.-K. Lam, "Dissipativity analysis and synthesis for discrete-time T-S fuzzy stochastic systems with time-varying delay," IEEE Transactions on Fuzzy Systems, vol. 22, no. 2, pp. 380-394, 2014.

[37] A. R. Lu, H. Wu, and J. Bai, "New delay-dependent robust stability criteria for uncertain neutral systems with mixed delays," Journal of the Franklin Institute, vol. 351, no. 3, pp. 13861399, 2014.

[38] H. Shen, S. Xu, J. Zhou, and J. Lu, "Fuzzy $H_{\infty}$ filtering for nonlinear Markovian jump neutral systems," International Journal of Systems Science, vol. 42, no. 5, pp. 767-780, 2011.

[39] S. He and F. Liu, " $L_{2}-L_{\infty}$ fuzzy control for Markov jump systems with neutral time-delays," Mathematics and Computers in Simulation, vol. 92, pp. 1-13, 2013.

[40] K. Gu, "An integral inequality in the stability problem of timedelay systems," in Proceedings of the 39th IEEE Conference on Decision and Control, vol. 3, pp. 2805-2810, Sydney, Australia, December 2000.

[41] H.-N. Wu and H.-X. Li, "New approach to delay-dependent stability analysis and stabilization for continuous-time fuzzy systems with time-varying delay," IEEE Transactions on Fuzzy Systems, vol. 15, no. 3, pp. 482-493, 2007. 


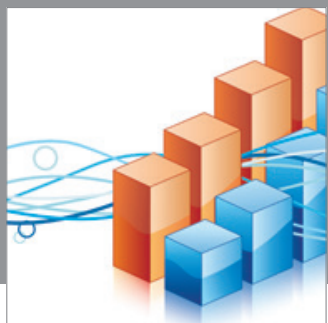

Advances in

Operations Research

mansans

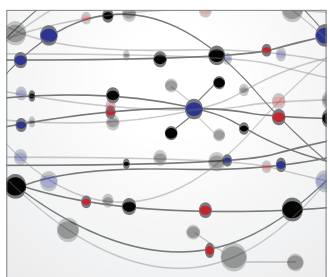

The Scientific World Journal
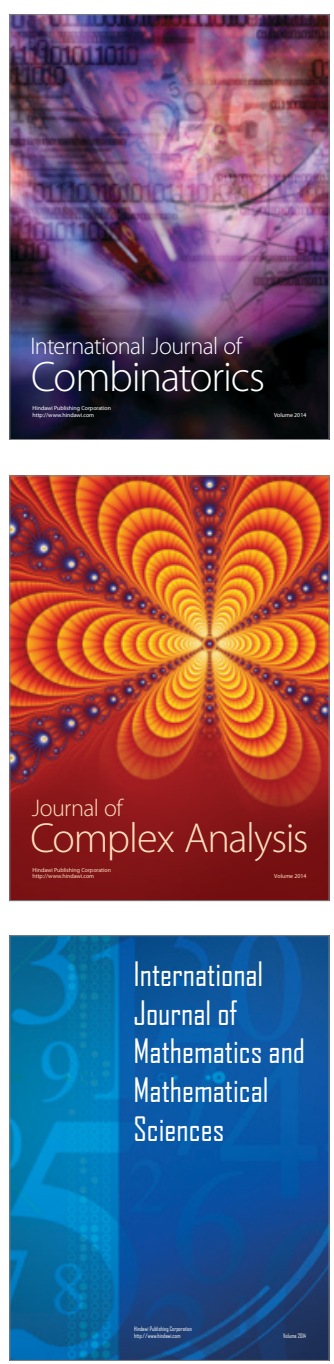
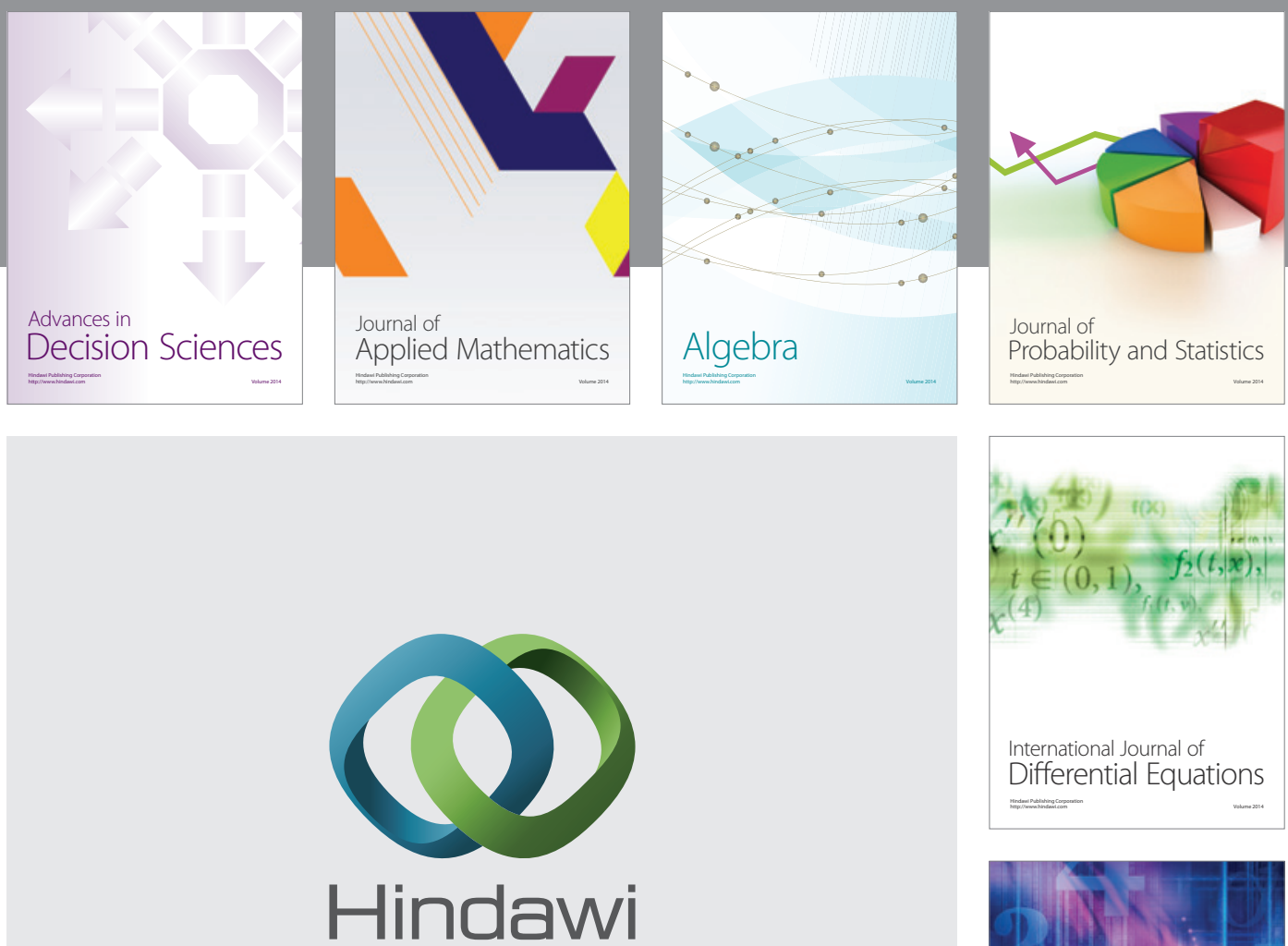

Submit your manuscripts at http://www.hindawi.com
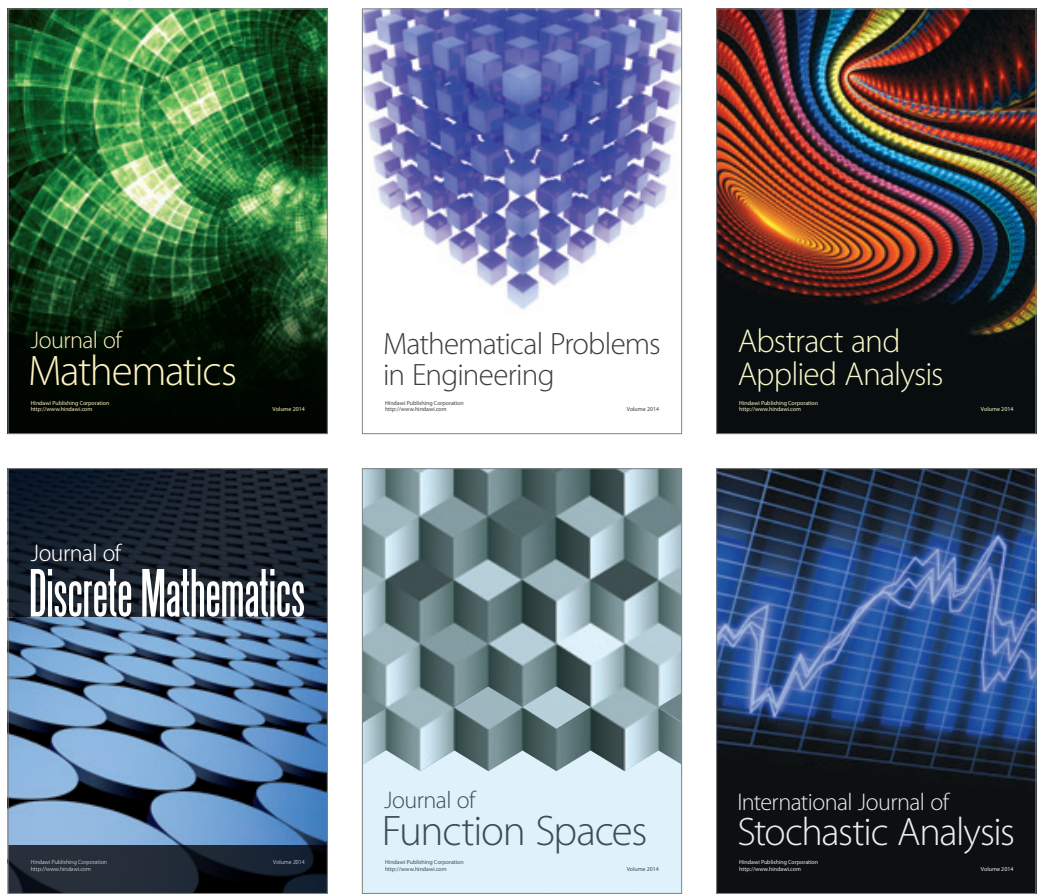

Journal of

Function Spaces

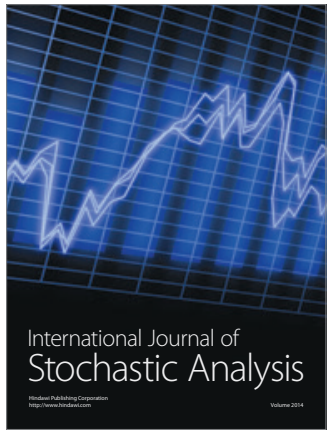

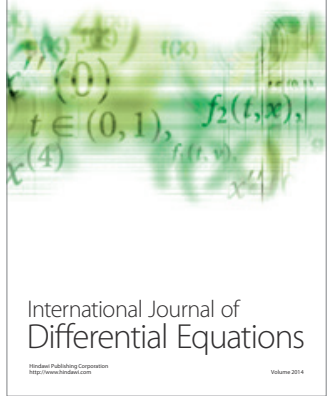
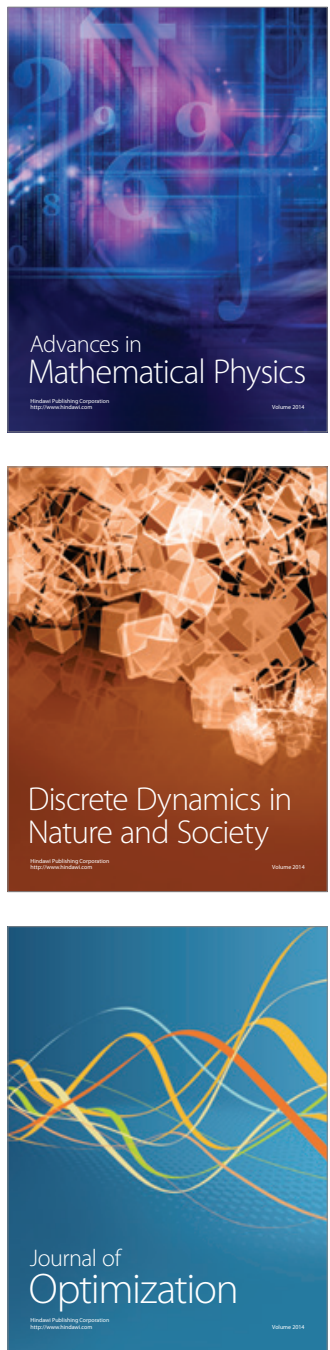